\title{
ReDO_DB: the repurposing drugs in oncology database
}

\author{
Pan Pantziarka ${ }^{1,2}$, Ciska Verbaanderd ${ }^{1,3}$, Vidula Sukhatme ${ }^{4}$, Rica Capistrano I', Sergio Crispino ${ }^{1}$, Bishal Gyawali ${ }^{1,5}$, \\ Ilse Rooman ${ }^{1,6}$, An MT Van Nuffel ${ }^{1}$, Lydie Meheus ${ }^{1}$, Vikas P Sukhatme ${ }^{4,7}$ and Gauthier Bouche ${ }^{1}$ \\ ${ }^{1}$ The Anticancer Fund, Brussels, 1853 Strombeek-Bever, Belgium \\ ${ }^{2}$ The George Pantziarka TP53 Trust, London, UK \\ ${ }^{3}$ Clinical Pharmacology and Pharmacotherapy, Department of Pharmaceutical and Pharmacological Sciences, KU Leuven, Leuven, Belgium \\ ${ }^{4}$ GlobalCures Inc., Newton, MA 02459 USA \\ ${ }^{5}$ Department of Medicine, Brigham and Women's Hospital, Harvard Medical School, Boston, MA 02115 USA \\ ${ }^{6}$ Oncology Research Centre, Vrije Universiteit Brussel, Brussels, Belgium \\ ${ }^{7}$ Emory University School of Medicine, Atlanta, GA 30322 USA
}

Correspondence to: Pan Pantziarka. Email: anticancer.org.uk@gmail.com

\section{Abstract}

Repurposing is a drug development strategy that seeks to use existing medications for new indications. In oncology, there is an increased level of activity looking at the use of non-cancer drugs as possible cancer treatments. The Repurposing Drugs in Oncology (ReDO) project has used a literature-based approach to identify licensed non-cancer drugs with published evidence of anticancer activity. Data from 268 drugs have been included in a database (ReDO_DB) developed by the ReDO project. Summary results are outlined and an assessment of clinical trial activity also described. The database has been made available as an online open-access resource (http://www.redo-project. $\underline{\mathrm{org} / \mathrm{db} /)}$.

Keywords: drug repurposing, repositioning, ReDO project, cancer drugs, online database

Published: 06/12/2018

Received: 27/09/2018

ecancer 2018, 12:886 https://doi.org/10.3332/ecancer.2018.886

Copyright: ( $@$ the authors; licensee ecancermedicalscience. This is an Open Access article distributed under the terms of the Creative Commons Attribution License (http://creativecommons.org/licenses/by/3.0), which permits unrestricted use, distribution, and reproduction in any medium, provided the original work is properly cited. 


\section{Introduction}

Drug repurposing, also known as repositioning, is a strategy that seeks new medical treatments from among existing licensed medications rather than from the development of new molecules (de novo drug development) [1]. Repurposing is by no means a new idea in medicine, indeed, many venerable and well-established drugs, for example, the beta-blocker propranolol, have been extensively repurposed many times in the past. However, as an explicit development strategy, repurposing is being increasingly pursued in a number of different disease areas [2-4]. Indeed, data from PubMed show that the number of publications related to drug repurposing or repositioning has increased exponentially since 2004 [5].

The Repurposing Drugs in Oncology (ReDO) project is an on-going collaborative project that has focused exclusively on the potential use of licensed non-cancer medications as sources of new cancer therapeutics [6]. While it is a common practice for new cancer medicines to be licensed for additional cancer indications after an initial license has been granted, a process that has been termed 'soft repurposing', the licensing of non-cancer medications as new treatments is relatively uncommon, hence this process has been termed 'hard repurposing' [7]. Indeed, there are very few examples in standard clinical practice of non-cancer drugs being moved into oncology, with thalidomide (multiple myeloma) and all-trans retinoic acid (acute promyelocytic leukaemia) being the best-known examples. In this sense, the ReDO project has focused exclusively on hard drug repurposing in oncology.

In contrast to de novo drugs, licensed medications may offer a number of advantages in terms of development [8]:

- Availability of pharmacokinetics, pharmacodynamics and posology data

- Knowledge of safety and toxicity, including rare adverse events

- Clinical experience derived from the original indications

- Widespread availability_particularly for drugs included in the WHO Essentials Medicines list (EML)

- Low cost-particularly for generic medications with multiple manufacturers

- Understanding of mechanisms of action and/or molecular targets

However, while these advantages may shorten the development period in comparison to unlicensed medications, particularly with respect to early phase toxicity trials, proving efficacy in any new indications remains a challenge. Despite the advantages that repurposed drugs may offer in terms of toxicity and cost, the single most important criterion by which treatments should be judged is efficacy. Of course, this also means that the medical community should judge the relative merits of repurposed versus new drugs without bias [9].

While much of the burgeoning interest in oncological repurposing is related to a few very high profile candidates, such as aspirin or metformin, there is indeed a wide range of non-cancer drugs which have some level of evidence in support of relevant anticancer activity $[10,11]$. This paper introduces ReDO_DB_a database of non-cancer drugs with evidence of anticancer activity that has been developed as part of the ReDO project. In addition to outlining the methodology and a selection of results from the database, it also gives details of the online open access publication of the database so that the data can be freely used by clinicians and researchers interested in developing specific repurposing projects.

\section{Methodology}

The ReDO project has adopted a literature-based methodology to identify non-cancer drugs with anticancer potential. The academic literature was actively scanned and potential repurposing candidates identified.

\section{Selection criteria}

Potential candidates must match the following criteria:

- The drug is currently licensed for non-cancer indications in at least one country in the world. Not included are:

- Existing cancer drugs, including cytotoxics, targeted agents or immunotherapeutics, (e.g. docetaxel, cyclophosphamide etc)

- Drugs withdrawn globally (e.g. phenformin) 
- Experimental medicines or previously shelved compounds (e.g. semagacestat, licofelone etc)

- Nutraceuticals (e.g. curcumin, resveratrol etc)

- The drug is the subject of one or more peer-reviewed publications showing a specific anticancer effect in one or more malignancies.

The evidence for anticancer effects could come from in vitro, in vivo or human research (as assessed by performing a PubMed search). In silico studies supported with in vitro or in vivo data were also included.

Drugs are included in the database if they fulfil the criteria above. In some cases, there may be indirect evidence to suggest that a drug may have anticancer activity because it has effects on an oncologically relevant pathway. However, if there is no explicit evidence of an effect-in other words, the evidence is purely mechanistic, then the drug is not included in the database.

\section{Data collected}

For each drug added to the database, we recorded its name (international non-proprietary name), synonyms (if relevant) and main approved indications. Each drug was also checked to see if it is available as a generic and whether it is included in the WHO List of Essential Medicines [12]. Multiple data sources were checked to assess whether a drug is available as a generic or is off-patent, although in some cases it was not possible to ascertain the current position with respect to patent protection. We also collected information on the type of research showing the anticancer activity of the drug: in vitro, in vivo and in humans.

Human data could include individual case reports, case series, epidemiological studies and clinical trials. Case reports were assessed using the following PubMed search terms: ('Case Reports' [Publication Type]) AND cancer AND <drug name>. Observational studies were assessed using the following PubMed search terms: ('Observational Study' [Publication Type]) AND Cancer AND <drug name>. Clinical data included published trial reports, of any phase, and existing clinical trial activity (as assessed by checking ClinicalTrials.gov, WHO ICTRP and OpenTrials registries). ReDO_DB was cross-referenced with DrugBank [13, 14] for additional analysis. Data were extracted on the Anatomical Therapeutic Chemical Classification System codes for each drug [15]. Additionally, DrugBank was used to extract data on the validated molecular targets for each drug. Note that these targets are not cancer-specific and as with the ReDO_DB data presented here, the data on molecular targets is a snapshot based on release 5.1.1 of DrugBank (release date 03 July 2018).

Finally, a search was performed to assess clinical trial activity by the drug. Three international clinical trial registries (ClinicalTrials.gov, WHO ICTRP and OpenTrials) were searched for each of the drugs on 16 August 2018. Only active late-stage oncology trials were included-that is trials flagged as Phase $2 / 3$, Phase 3 or Phase $3 / 4$. Each trial was manually assessed to remove trials in which the drugs were being used for their original indication, for example, trials in which licensed antiemetics were being assessed in new cancer or in combination treatments with other antiemetics. For each trial, the following information were collected: drug tested, countries (and continent), sponsor and cancer type.

\section{Results}

\section{Drugs in the ReDO database}

We found a total of 268 drugs that met our selection criteria. In order to maximise the utility of the database, an open access version is made available online via the website of the ReDO project (www.redo-project.org/db). The online version will be periodically updated so that as new drugs are added to the database, or new data become available for existing drugs on the database, the information can be made available to the oncology community. Additions or amendments may also be proposed via an online contact form, enabling other members of the oncology community to contribute to the development of the database. The online database has also been structured in a format to facilitate easy data-mining, spidering or simple cut and paste to maximise accessibility.

The following results summarise the data in the ReDO_DB as of 16 August 2018 when 268 drugs were included. Summary statistics are shown in Table 1. 
It should be noted that $25 \%$ of drugs meet multiple favourable criteria in that they are on the WHO List of Essential Medicines, are off-patent and have some form of human evidence of anticancer effects.

The complete list of drugs is included in the supplementary data.

Repurposing candidates come from a wide range of areas of medicine. Using the Anatomical Therapeutic Chemical Classification System, we can assess the sources of ReDO_DB drugs, as shown in Table 2. Note that some drugs are included in multiple ATC categories, and therefore the total is greater than the number of drugs in the ReDO dataset.

Data on molecular targets are shown in Table 3.

\section{Late stage oncology trials}

In all 190 relevant late-stage trials were identified. Data from this analysis are shown in Table 4.

The characteristics of the 190 trials are summarised in Table 5. Figure 1 shows a map of the countries where the trials have been or are being conducted. A small number of drugs are currently the subject of intense clinical trial activity (i.e. 10 or more active late-stage trials) and should be considered to be well advanced in terms of a 'repurposing drugs pipeline' in oncology. In terms of clinical trial sponsorship, the data show that the very few trials have a commercial sponsor-less than $4 \%$ of trials in this dataset.

\section{Discussion}

Data from ReDO_DB show that there are in fact a large number of non-cancer drugs with published evidence of anticancer effects. The majority $(73 \%)$ have some evidence of anticancer effects from case reports, observational studies or clinical trials. Furthermore, the majority (84\%) are off-patent, and $32 \%$ are included in the WHO EML. The number of drugs which have human data, are off-patent and included in the WHO EML is 67 , representing $25 \%$ of the total database. This represents a promising pipeline of potential new treatments in oncology. It is indeed encouraging that there are currently just under 200 late-stage clinical trials investigating the use of these drugs in oncology. However, given the high unmet needs in paediatric oncology, it is not so encouraging to note that only $6 \%$ of these trials are in childhood cancers.

Table 1. Summary statistics from ReDO_DB as of 15 August 18.

\begin{tabular}{|l|c|c|c|c|c|}
\hline \multicolumn{1}{|c|}{ Drugs ... } & Yes & $\%$ & No & $\%$ & Total \\
\hline Are included in WHO List of Essential Medicines? & 87 & 32 & 181 & 68 & 268 \\
\hline Are off-patent? § & 226 & 84 & 35 & 13 & 268 \\
\hline Are supported by in vitro evidence? & 264 & 99 & 4 & 1 & 268 \\
\hline Are supported by in vivo evidence? & 247 & 92 & 21 & 8 & 268 \\
\hline Are supported by case reports? & 86 & 32 & 182 & 68 & 268 \\
\hline Are supported by observational studies? & 36 & 13 & 232 & 87 & 268 \\
\hline Have been/Are being tested in clinical trials? & 178 & 66 & 90 & 34 & 268 \\
\hline Have trial report(s) published? & 113 & 63 & 65 & 37 & 178 \\
\hline Are supported by human data? ${ }^{*}$ & 194 & 72 & 74 & 28 & 268 \\
\hline Are WHO + Off-patent + Human data?* & 67 & 25 & 201 & 75 & 268 \\
\hline
\end{tabular}

${ }^{*}$ At least one case report, observational study or clinical trial.

$\S$ It was not possible to ascertain the patent position for $3 \%$ of drugs. 
Table 2. Number of drugs by top-level ATC category.

\begin{tabular}{|l|c|}
\hline \multicolumn{1}{|c|}{ ATC Level 1 } & Drugs \\
\hline Cardiovascular System & 56 \\
\hline Nervous System & 49 \\
\hline Alimentary Tract and Metabolism & 39 \\
\hline Musculo-Skeletal System & 31 \\
\hline Antiinfectives for Systemic Use & 26 \\
\hline Dermatologicals & 23 \\
\hline Genito Urinary System and Sex Hormones & 23 \\
\hline Sensory Organs & 22 \\
\hline Antiparasitic Products, Insecticides and Repellents & 20 \\
\hline Blood and Blood-Forming Organs & 16 \\
\hline Antineoplastic and Immunomodulating Agents & 12 \\
\hline Respiratory System & 11 \\
\hline Systemic Hormonal Preparations, Excl. Sex Hormones and Insulins & 4 \\
\hline Various & 4 \\
\hline
\end{tabular}

Table 3. Molecular targets included in ReDO drugs.

\begin{tabular}{|l|c|}
\hline \multicolumn{1}{|c|}{ Item } & \\
\hline ReDO drugs included in DrugBank & \\
\hline ReDO drugs with targets in DrugBank & 263 \\
\hline Total targets identified in all ReDO drugs & 252 \\
\hline Number of unique targets in ReDO drugs & 1201 \\
\hline Average targets per drug & 660 \\
\hline
\end{tabular}

*Five drugs approved for use outside of the USA and the EU are not currently included in DrugBank.

Table 4. ReDO drugs included in late-phase clinical trials.

\begin{tabular}{|l|c|}
\hline \multicolumn{1}{|c|}{ Item } & \\
\hline Number of relevant late-stage trials & 190 \\
\hline Number of unique drugs & 72 \\
\hline Number of drugs with 5 or more trials & 11 \\
\hline Number of drugs with 10 or more trials & 6 \\
\hline
\end{tabular}


Table 5. Characteristics of the 190 trials registered with one of the 72 drugs of the ReDO_DB tested in clinical trials.

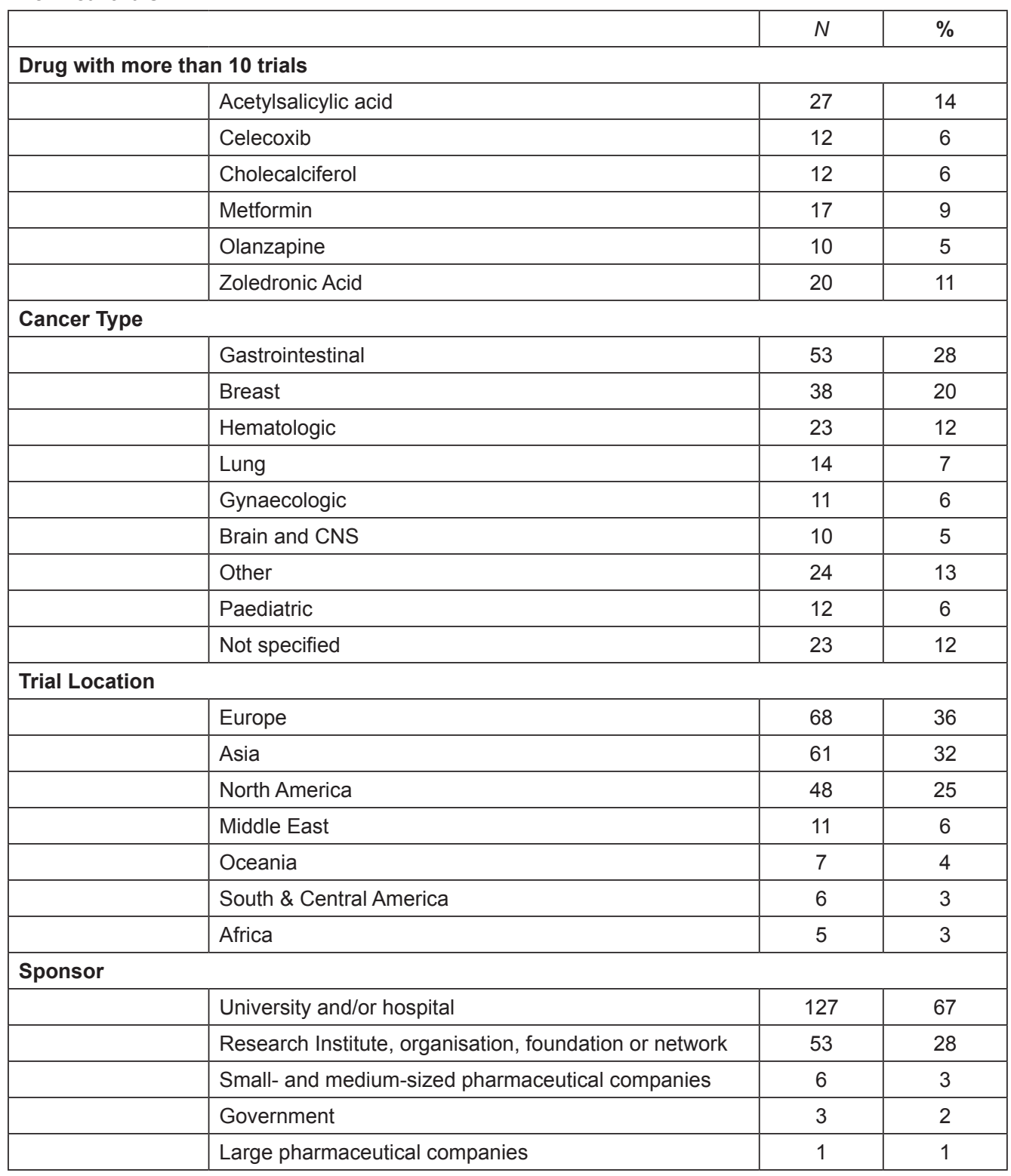




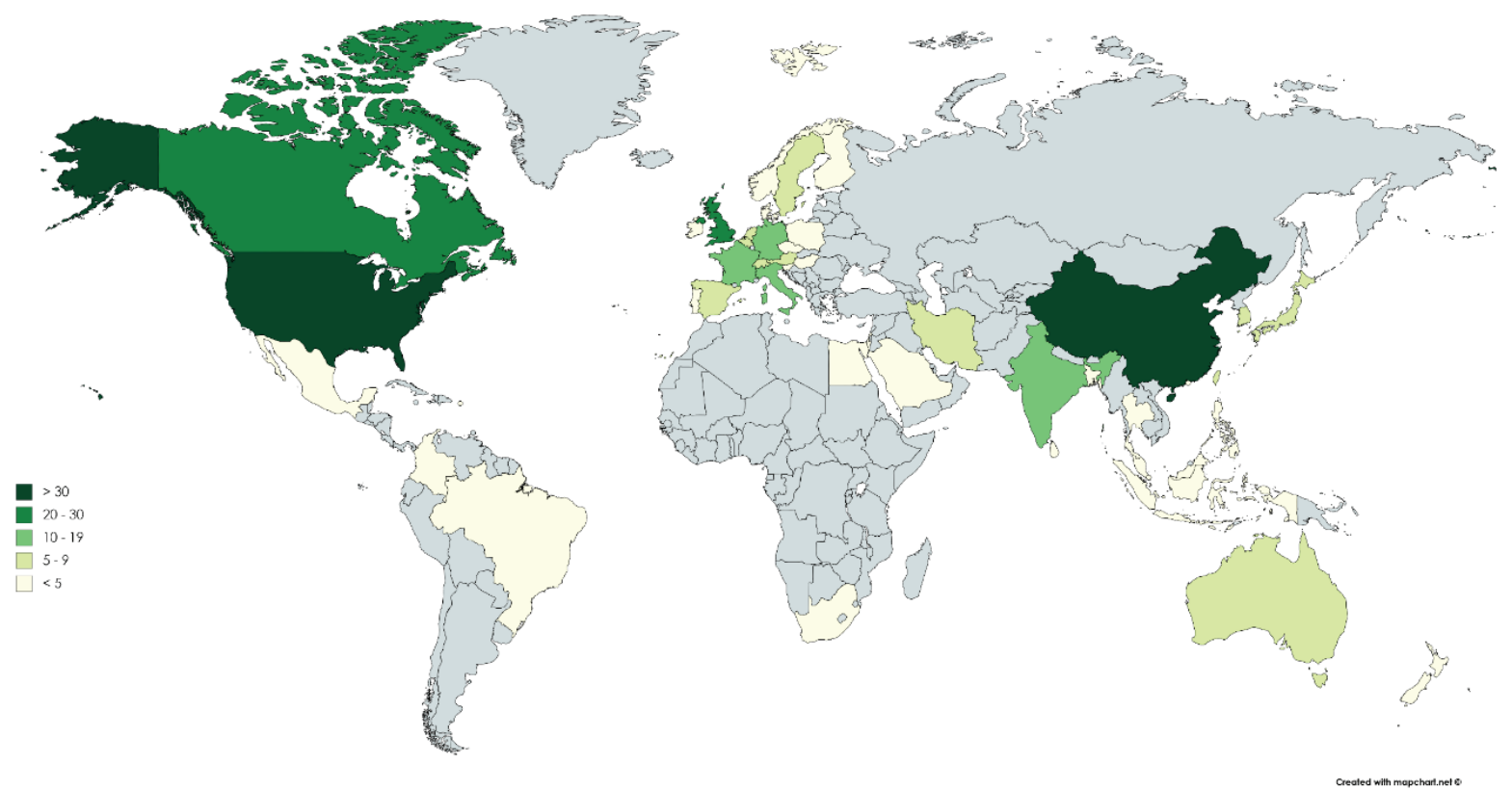

Figure 1. World map showing the number of late-stage trials of the drugs in the ReDO_DB per country.

In terms of molecular drug targets, it is likely that the number of targets reported in Table 3 is an underestimate based on analysis by Mestres et al which showed that for a large panel of drugs, the average number of target proteins per drug is 6.3 if additional data sources to DrugBank are accessed [16].

The ReDO_DB has both strengths and limitations. One strength is that the database has been built prospectively over the last 5 years, allowing us to manually curate and validate each drug. We have also benefited from the help of a large network of individuals interested in drug repurposing in oncology. However, we acknowledge that at any given time, the database is incomplete in that new data are published and new candidates emerge. Currently, new entries to the database are added regularly and it is hoped that with the database becoming publicly available, a crowdsourcing effect may help to increase the level of completeness of the database.

ReDO_DB does not include drugs that have solely in silico evidence of a possible role in oncology. While our criteria depend on biological data (in vitro or in vivo) for the inclusion of a candidate drug in the database, we acknowledge the value of in silico work as it is often the first method to suggest a brand new use for an existing drug [17]. One limitation of the database is that it does not include existing cancer drugs which represent a large source of repurposing opportunities. However, virtually all cancer drugs are possible candidates for repurposing in other cancer types and listing them in the ReDO_DB would not be of any added value. We also have not included approved vaccines in the database but we are considering doing so, building on the case of Bacillus Calmette-Guérin [18] and looking at recent evidence in support of the repurposing of influenza [19] or cholera vaccine [20].

The inclusion of a number of drugs was problematic in that they are already licensed for use in oncology for symptomatic relief (e.g. aprepitant to control chemotherapy-associated nausea and vomiting) or cancer-related events (e.g. zoledronate or ibandronate for reduction of bone-related events in advanced malignancies). In the former case, drugs are included if there is data to suggest that there is specific anticancer activity independent of the existing licensed indication. With the cases of zoledronate and ibandronate, the issue is complicated in that there is some existing 'off-label' use of the drugs for specific anticancer effects. However, as this use is currently off-label, the drugs have been included in the database. 
While the inclusion of new drugs in the database is fairly straightforward and is based on the criteria outlined previously, the removal of repurposing candidates is more complex. Failure of a repurposing candidate in a clinical trial is insufficient grounds for removal as the drug may still be active in a different cancer, treatment setting, drug combination or dose. Where a drug has been included based solely on published preclinical work that is later shown to be fraudulent, then removal would be warranted if there is no other supporting evidence. However, the clearest case for removal is when a repurposed drug becomes licensed as a new cancer treatment-in that case, the drug moves into the 'soft repurposing' category for further development in other cancer indications and will be removed from the ReDO_DB.

With such a broad range of drugs and so many validated molecular targets, discussion of general mechanisms of action and research priorities is not possible. The vast majority of these drugs do not induce cancer cell cytotoxicity but instead act systemically on the host, alter the immune response or else affect aspects of the tumour microenvironment. These effects may provide therapeutic benefit to cancer patients when used in combination with existing treatments.

The main challenge will be to test these hypotheses to ultimately find cancer indications, if any, for each candidate. For drugs that are already well-studied (e.g. disulfiram or nelfinavir), a meticulous analysis of the data available is needed to identify the most relevant clinical trials to be conducted. For less well-studied drugs (e.g. fasudil or trimetazidine), more research may first be needed to explore and guide the possible future of those drugs. Another possible source of indications for some candidates may be in precision oncology efforts [7].

A number of online cancer-related drug repurposing databases already exist, including DRUGSURV [21], DeSigN [22] and IMPACT [23]. However, these databases are primarily designed to facilitate the discovery of new repurposing candidates using different data sources and algorithmic techniques. In contrast, ReDO_DB presents a curated list of repurposing candidates and a summary of the types of data sources supporting the inclusion of the drug in the database. Outside of oncology, the PDE3 (Prescribable Drugs with Efficacy in Experimental Epilepsies) [2] is an example of a database similar in scope and intention to ReDO_DB.

There is clearly a scope to increase the value of the database in the future by the inclusion of additional data fields. One possibility is to include an indication of the strength of evidence for each of the drugs in addition to showing the range (in vitro, in vivo etc) of evidentiary sources. Other enhancements may also be proposed by users of the database in the future.

\section{Conclusion}

The results outlined in this paper are generally positive in showing both a growth of interest in drug repurposing, a wide range of candidates for repurposing in oncology and 190 late-stage clinical trials. However, it is also true that there are numerous obstacles in the path to successful repurposing. Because many of the repurposing candidates are generic drugs, (84\% in the ReDO dataset), commercial funding of clinical trials is normally not an option. Indeed, the data in Table 5 show that only 7 of 190 trials were sponsored by pharmaceutical companies.

There are significant costs associated with carrying out large Phase III efficacy trials_repurposing trials are therefore at a disadvantage in that they must rely on state or philanthropic sources of funding. Indeed, there is even some evidence to suggest that for an institution there is a financial benefit from running a commercial trial (i.e. per patient net income) compared to a non-commercial trial (i.e. per patient net cost) $[24,25]$. In some cases, there may be commercial support for repurposing trials if a commercial sponsor is looking to increase efficacy or expand an indication for an on-patent drug by combining with a repurposing candidate. There may also be cases where insurers or other payers may wish to fund studies that have the potential to reduce cancer recurrence rates or other interventions designed to reduce their costs. Finally, the costs of studies using repurposed drugs may fall if suitable biomarkers are used to stratify patients for enrolment who are most likely to benefit, thereby reducing patient numbers required to show an effect.

Here again repurposing faces a financial obstacle in that there are costs associated with licensing a drug for a new indication (technically, a label extension). It is also the case that there are regulatory restrictions on who can apply for a label extension-therefore, it is important to make the case for a 'public benefit label extension' process so that we can move clinically-proven repurposing from 'off-label' to 'on-label' treatments [26]. In time, we hope that we will see the ReDO_DB shrink as repurposed drugs are licensed for new cancers indications, at which point they will be removed from the database. 


\section{Acknowledgments}

This work would not be as extensive without the contribution of multiple collaborators, researchers and clinicians who share our interest in drug repurposing. We are grateful to Richard Kast, Andrew Wilcock, Michael Jameson, Lars Søraas, Nicolas André, Albrecht Reichle, Richard Sullivan, Bjørn Tore Gjertsen, Peter Nygren, our colleagues at the Anticancer Fund and the team at ecancermedicalscience ... and many others who have shared their findings with us to make this list as complete as possible.

\section{Competing interests}

The authors declare that they have no competing interests. All the authors are associated with not for profit organisations that aim to repurpose drugs for oncology treatments. VPS is also a scientific advisory board member of Berg Health and Mitra Biotech.

\section{References}

1. Langedijk J, Mantel-Teeuwisse AK, and Slijkerman DS, et al (2015) Drug repositioning and repurposing: terminology and definitions in literature Drug Discov Today 20(8) 1027-1034 https://doi.org/10.1016/j.drudis.2015.05.001 PMID: 25975957

2. Sivapalarajah S, Krishnakumar M, and Bickerstaffe $\mathrm{H}$, et al (2018) The prescribable drugs with efficacy in experimental epilepsies (PDE3) database for drug repurposing research in epilepsy Epilepsia 59(2) 492-501 https://doi.org/10.1111/epi.13994 PMID: 29341109

3. Mercorelli B, Palù G, and Loregian A (2018) Drug repurposing for viral infectious diseases: how far are we? Trends Microbiol 26(10) 865-876 https://doi.org/10.1016/j.tim.2018.04.004 PMID: 29759926

4. Kakkar AK, Singh H, and Medhi B (2018) Old wines in new bottles: repurposing opportunities for Parkinson's disease Eur $J$ Pharmacol 830 115-127 https://doi.org/10.1016/j.ejphar.2018.04.023 PMID: 29689247

5. Shah RR and Stonier PD (2018) Repurposing old drugs in oncology: opportunities with clinical and regulatory challenges ahead J Clin Pharm Ther https://doi.org/10.1111/jcpt.12759

6. Pantziarka P, Bouche G, and Meheus L, et al (2014) The repurposing drugs in oncology (ReDO) project Ecancermedicalscience 8442 https://doi.org/10.3332/ecancer.2014.485 PMID: 25075216 PMCID: 4096030

7. Pantziarka P, Bouche G, and André N (2018) 'Hard’ Drug Repurposing For Precision Oncology: The Missing Link? Front Pharmacol 9(June) 637 https://doi.org/10.3389/fphar.2018.00637

8. Pantziarka P, Bouche G, and Meheus L, et al (2015) Repurposing drugs in your medicine cabinet: untapped opportunities for cancer therapy? Future Oncol 11(2) 181-184 https://doi.org/10.2217/fon.14.244 PMID: 25591833

9. Gyawali B, Pantziarka P, and Crispino S, et al (2018) Does the oncology community have a rejection bias when it comes to repurposed drugs? Ecancermedicalscience 12 10-14 https://doi.org/10.3332/ecancer.2018.ed76

10. Bouche G, Pantziarka $P$, and Meheus $L$ (2017) Beyond aspirin and metformin: the untapped potential of drug repurposing in oncology Eur J Cancer 72(72) S121-S122 https://doi.org/10.1016/S0959-8049(17)30479-3

11. Pantziarka P, Sukhtame V, and Meheus L, et al (2017) Repurposing non-cancer drugs in oncology - how many drugs are out there? bioRxiv 1

12. World Health Organization (WHO) (n.d.) WHO model lists of essential medicines [http://www.who.int/medicines/publications/essentialmedicines/en/] Date accessed: 20/08/18 
13. Wishart DS, Knox C, and Guo AC, et al (2008) DrugBank: a knowledgebase for drugs, drug actions and drug targets Nucleic Acids Res 36(Database Issue) D901-D906 https://doi.org/10.1093/nar/gkm958 PMCID: 2238889

14. Wishart DS, Feunang YD, and Guo AC, et al (2018) DrugBank 5.0: a major update to the DrugBank database for 2018 Nucleic Acids Res 46(D1) D1074-D1082 https://doi.org/10.1093/nar/gkx1037 PMCID: $\underline{5753335}$

15. World Health Organization (2003) The anatomical therapeutic chemical classification system Oslo, Norway: WHO

16. Mestres J, Gregori-Puigjané E, and Valverde S, et al (2008) Data completeness--the Achilles heel of drug-target networks Nature Biotechnol 26(9) 983-984 https://doi.org/10.1038/nbt0908-983

17. Liu Z, Fang $\mathrm{H}$, and Reagan $\mathrm{K}$, et al (2013) In silico drug repositioning: what we need to know Drug Discov Today 18(3-4) 110-115 https://doi.org/10.1016/j.drudis.2012.08.005

18. Herr HW and Morales A (2008) History of bacillus Calmette-Guerin and bladder cancer: an immunotherapy success story J Urol 179(1) 53-56 https://doi.org/10.1016/j.juro.2007.08.122

19. Tai L-H, Zhang J, and Scott KJ, et al (2013) Perioperative influenza vaccination reduces postoperative metastatic disease by reversing surgery-induced dysfunction in natural killer cells Clin Cancer Res 19(18) 5104-5115 https://doi.org/10.1158/10780432.CCR-13-0246 PMID: 23881927

20. Ji J, Sundquist J, and Sundquist K (2018) Association between post-diagnostic use of cholera vaccine and risk of death in prostate cancer patients Nature Commun 9(1) 2367 https://doi.org/10.1038/s41467-018-04814-4

21. Amelio I, Gostev M, and Knight RA, et al (2014) DRUGSURV: a resource for repositioning of approved and experimental drugs in oncology based on patient survival information Cell Death Dis 5(2) e1051 https://doi.org/10.1038/cddis.2014.9 PMID: 24503543 PMCID: $\underline{3944280}$

22. Lee BKB, Tiong KH, and Chang JK, et al (2017) DeSigN: connecting gene expression with therapeutics for drug repurposing and development BMC Genomics 18(Suppl 1) 934 https://doi.org/10.1186/s12864-016-3260-7 PMID: 28198666 PMCID: 5310278

23. Hintzsche JD, Yoo M, and Kim J, et al (2018) IMPACT web portal: oncology database integrating molecular profiles with actionable therapeutics BMC Med Genomics 11(Suppl 2) 4-9 https://doi.org/10.1186/s12920-018-0350-1

24. Liniker E, Harrison M, and Weaver JMJ, et al (2013) Treatment costs associated with interventional cancer clinical trials conducted at a single UK institution over 2 years (2009-2010) Br J Cancer 109(8) 2051-2057 https://doi.org/10.1038/bjc.2013.495 PMID: 24064969 PMCID: $\underline{3798949}$

25. Mañes-Sevilla M, Romero-Jiménez R, and Herranz-Alonso A, et al (2018) Drug cost avoidance in clinical trials of breast cancer J Oncol Pharm Pract 1078155218775193 https://doi.org/10.1177/1078155218775193 PMID: 29792122

26. Verbaanderd C, Meheus L, and Huys I, et al (2017) Repurposing drugs in oncology: next steps Trends Cancer 3(8) 543-546 https://doi.org/10.1016/j.trecan.2017.06.007 PMID: 28780930 


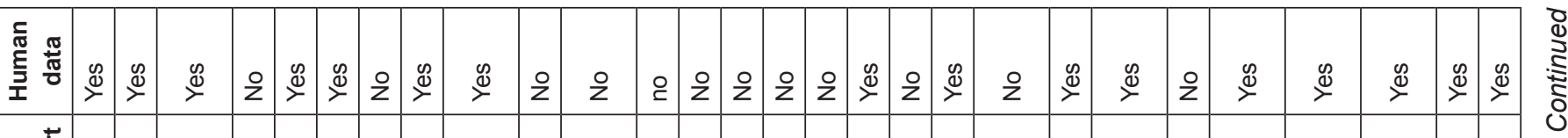

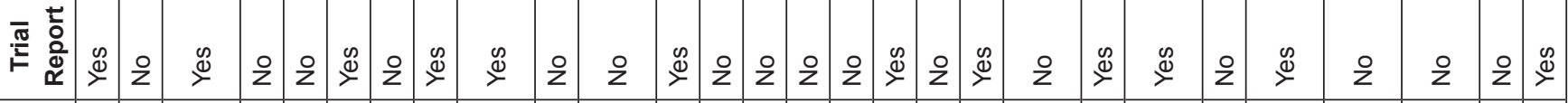

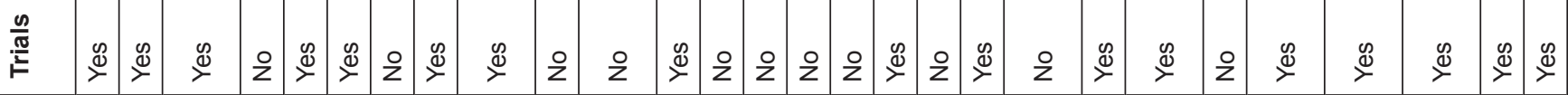

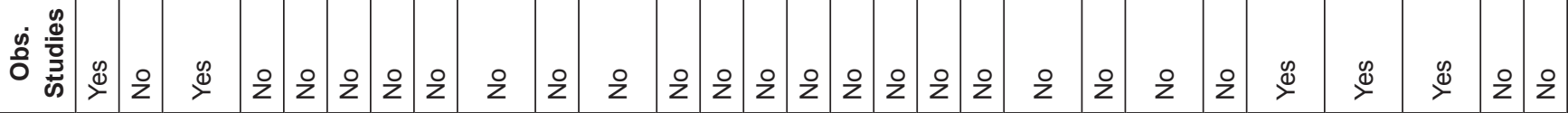

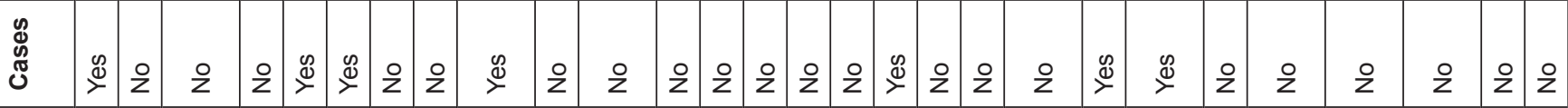

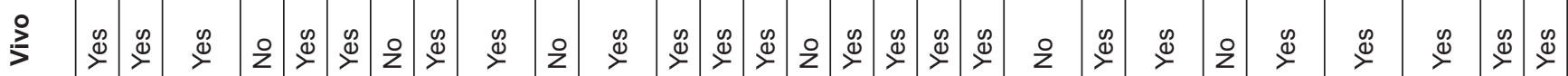

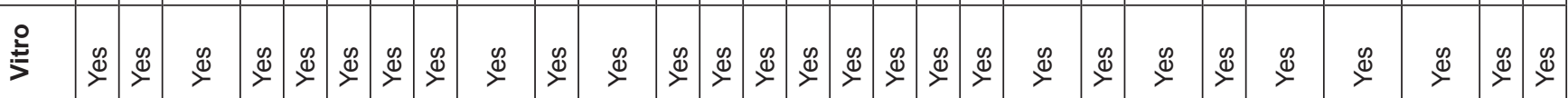

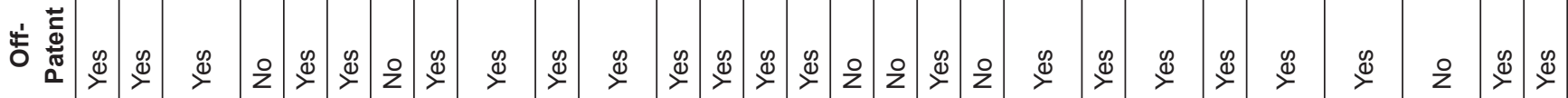

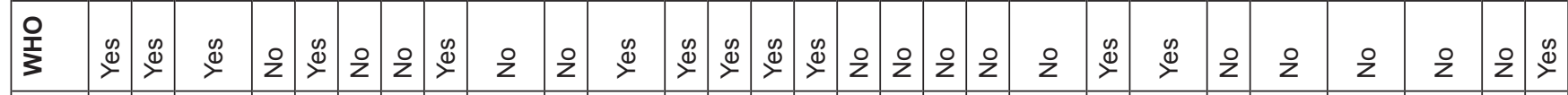

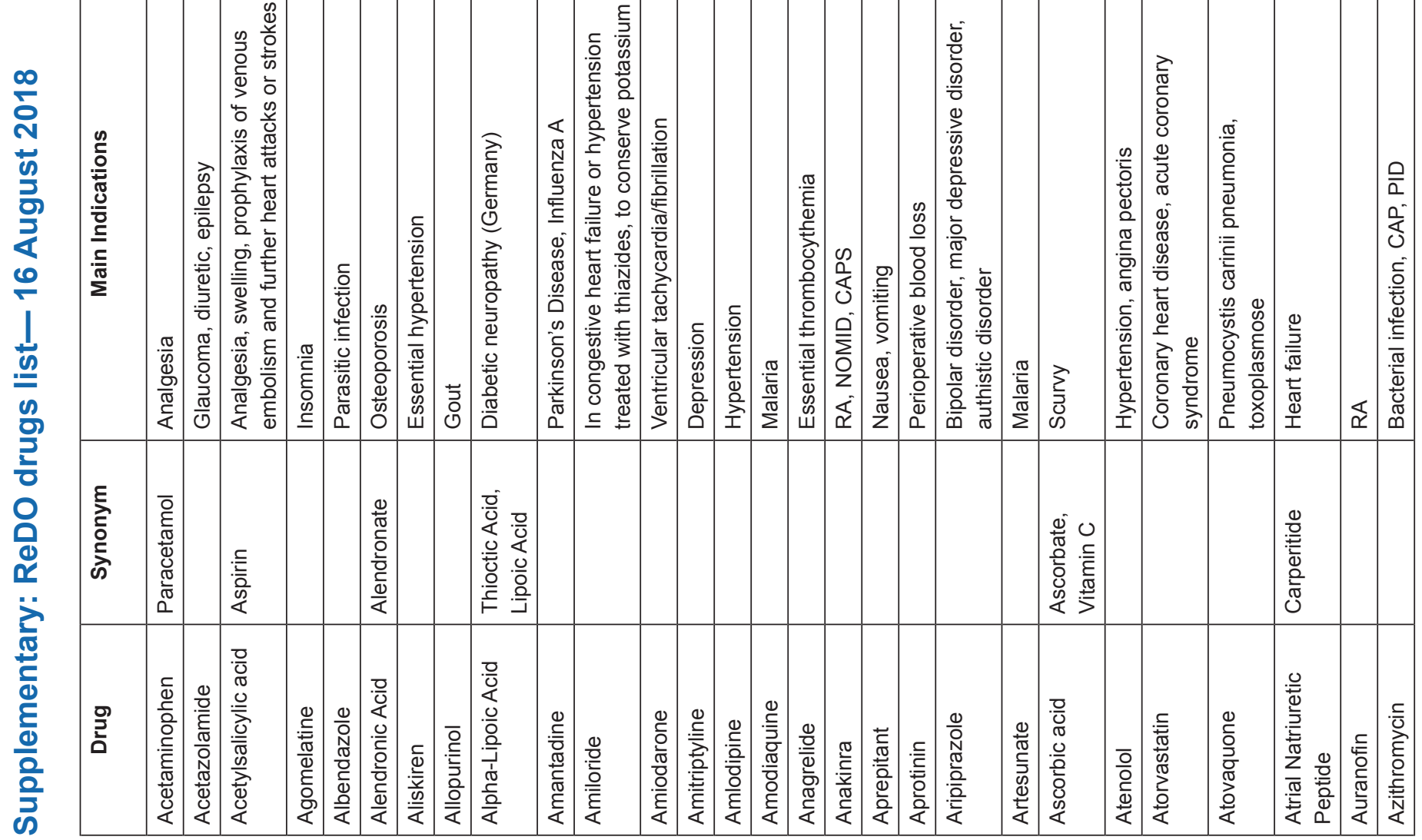




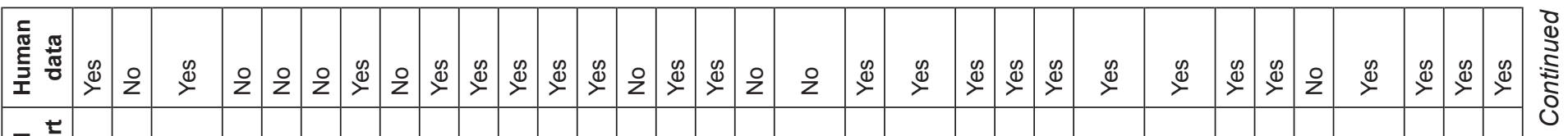

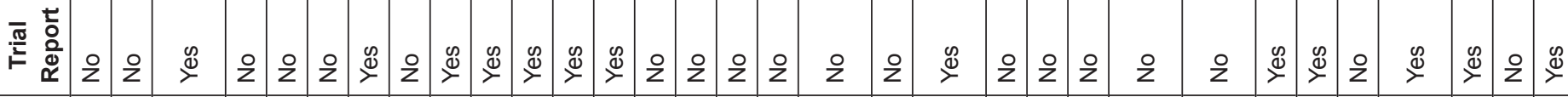

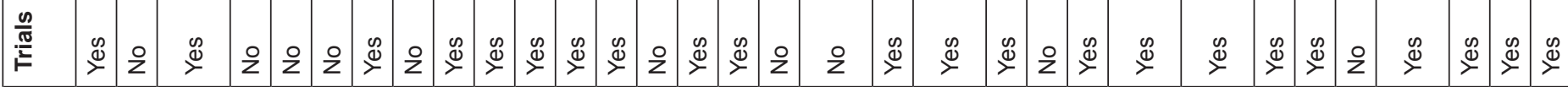

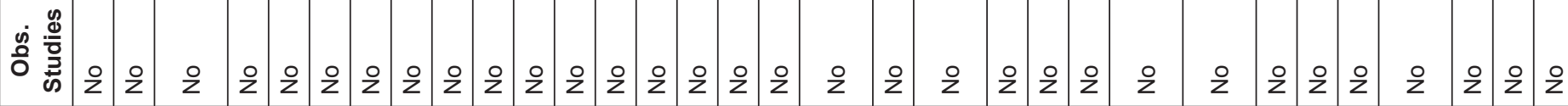

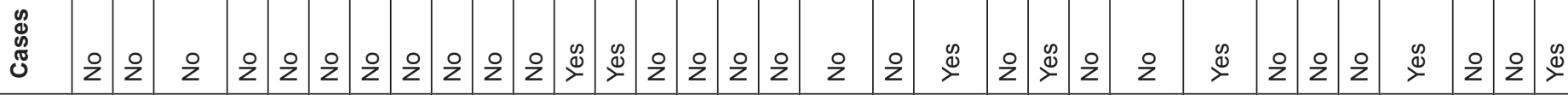

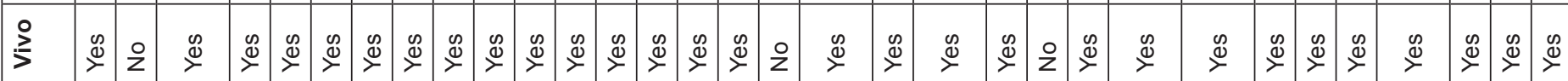

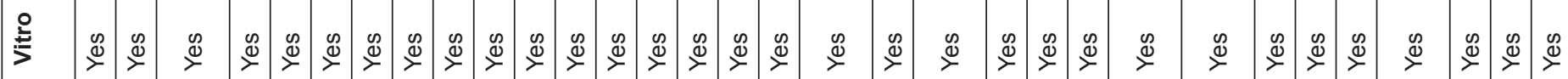

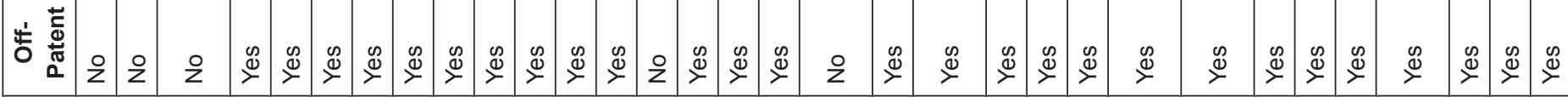

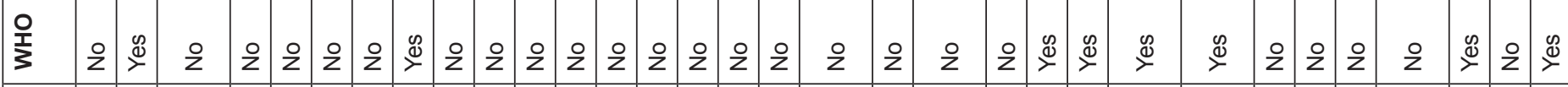

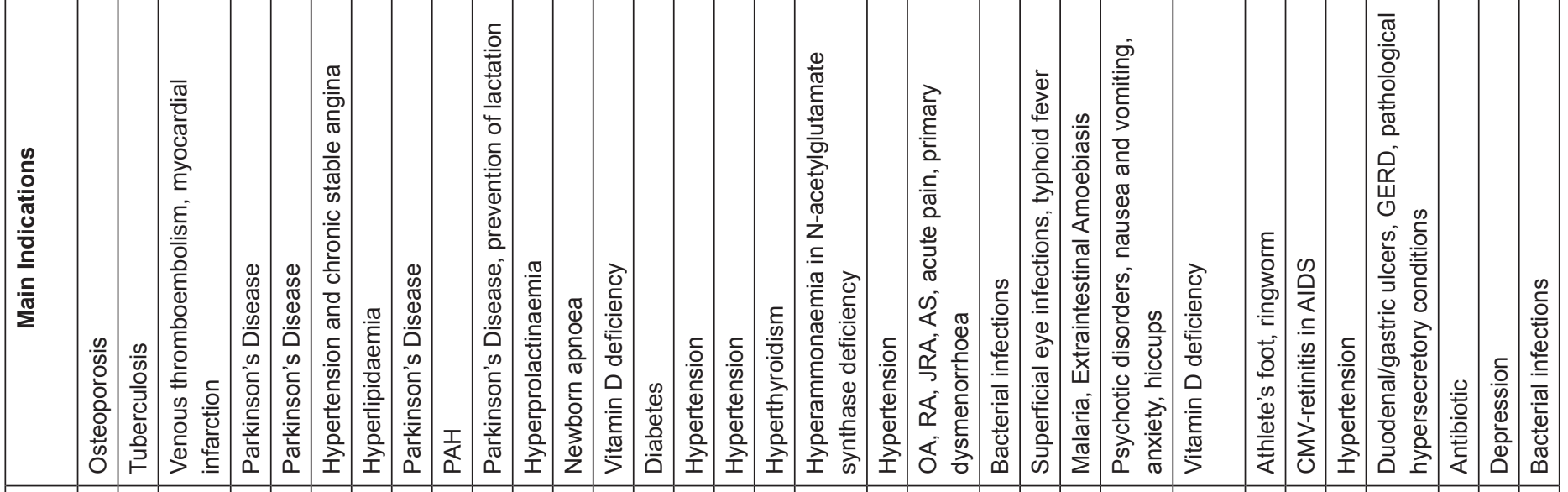

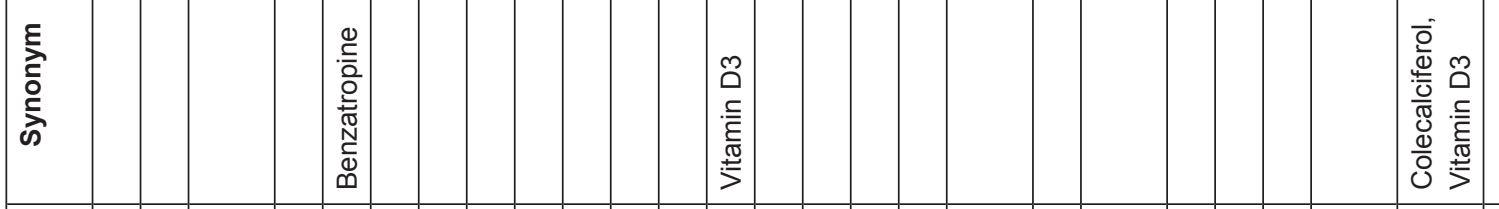

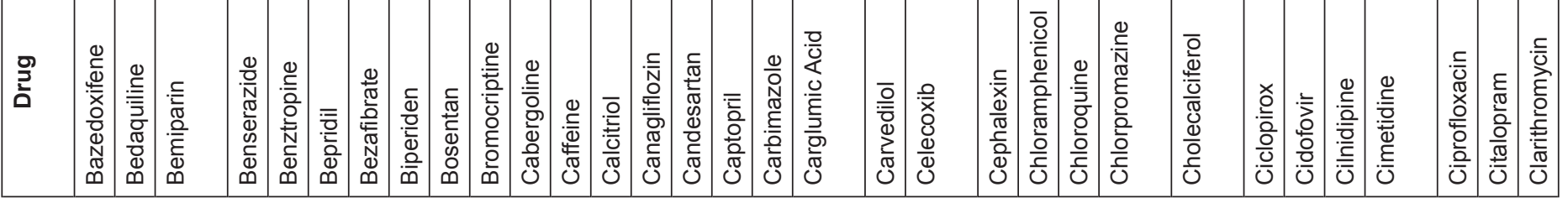




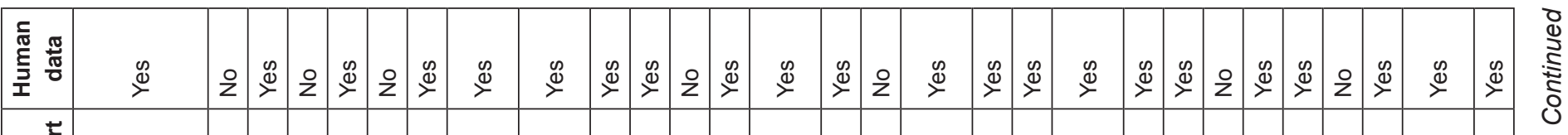

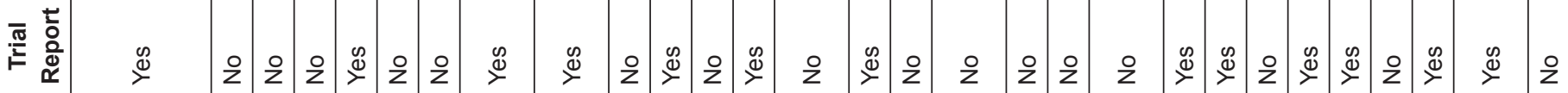

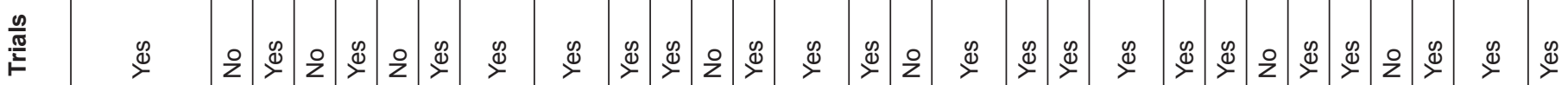

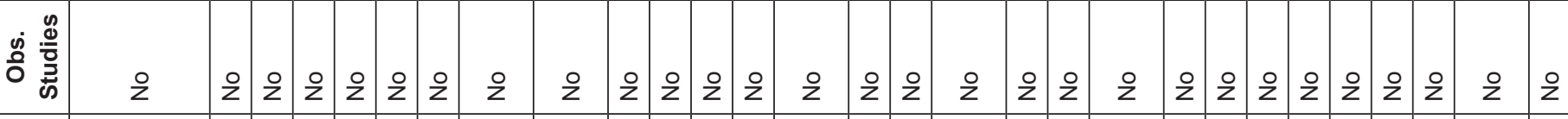

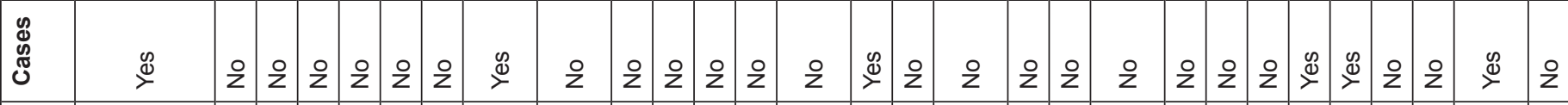

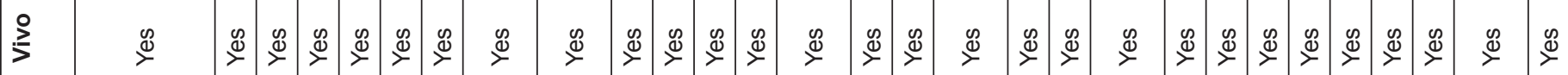

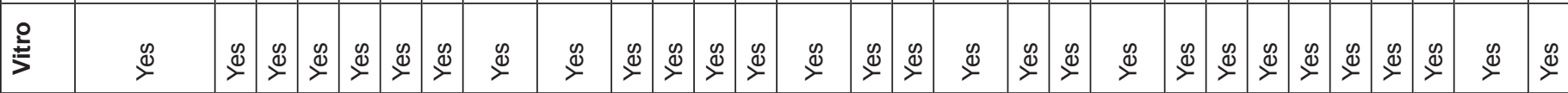

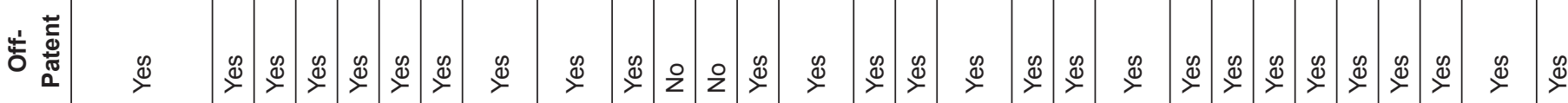

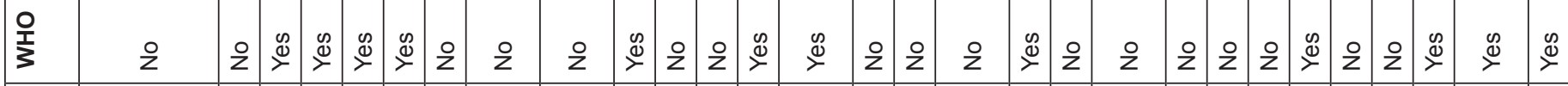

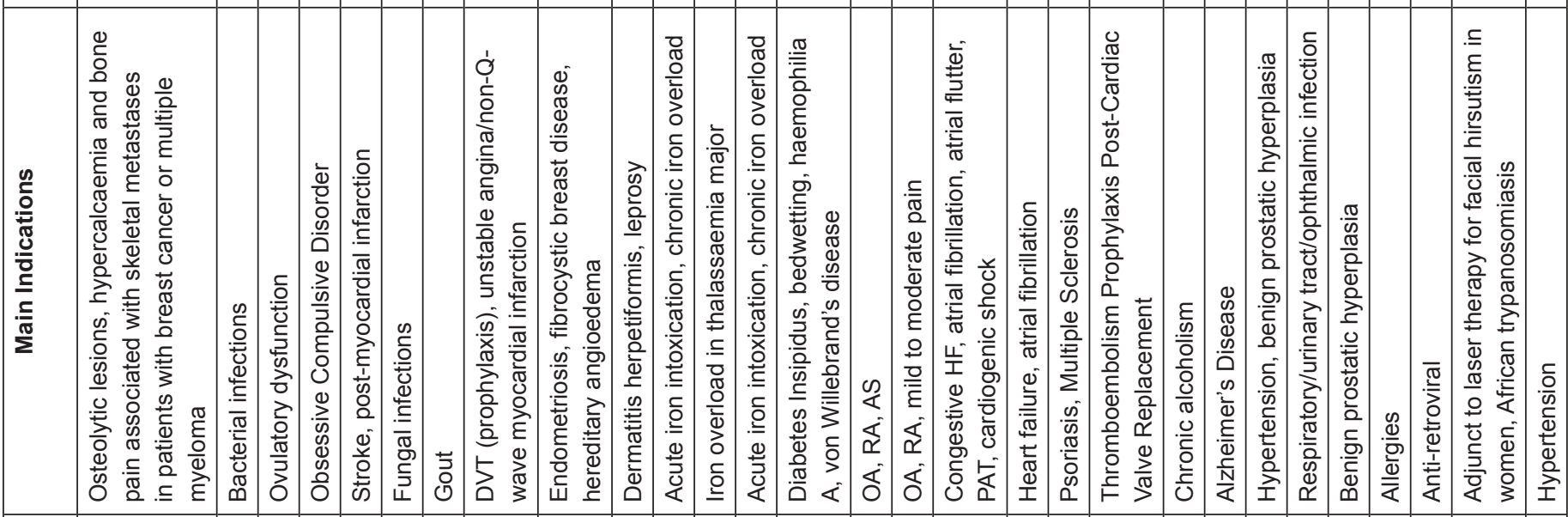

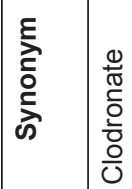

$\sum_{\text {咅 }}^{\circ}$

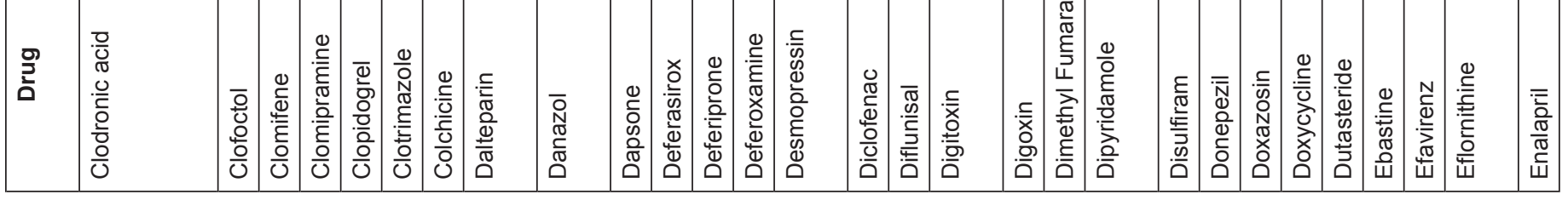




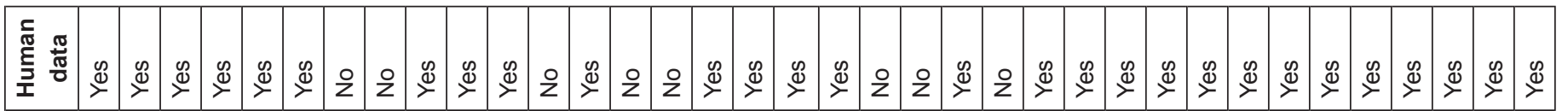

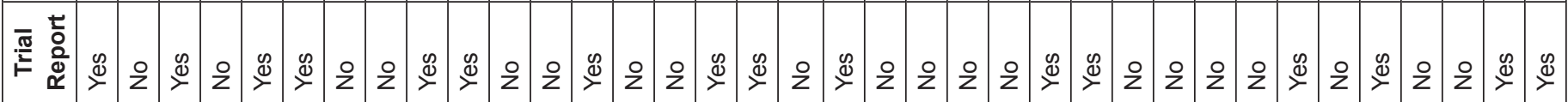

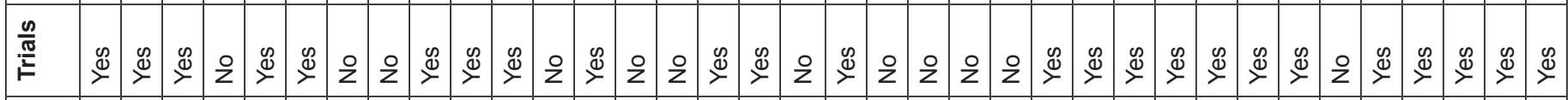

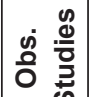

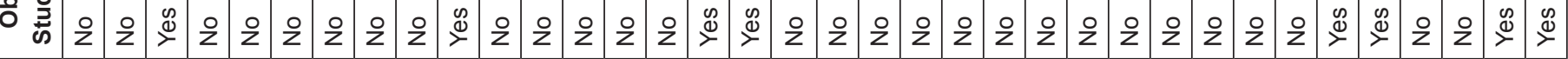

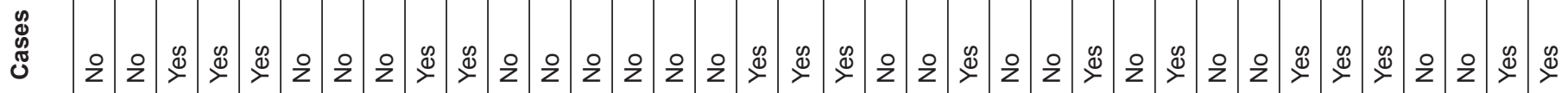

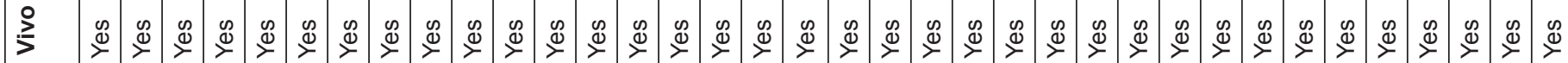

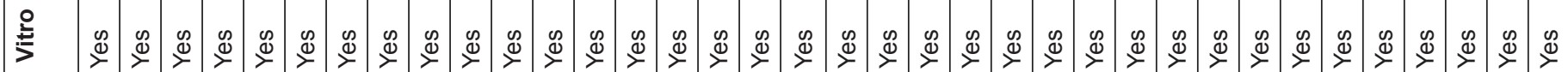

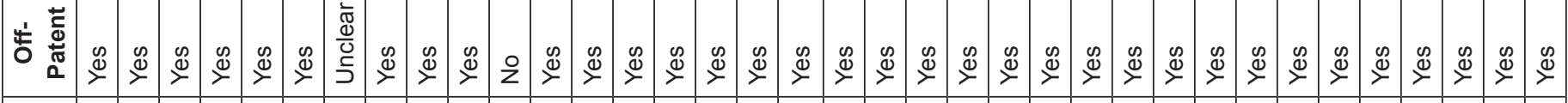
울
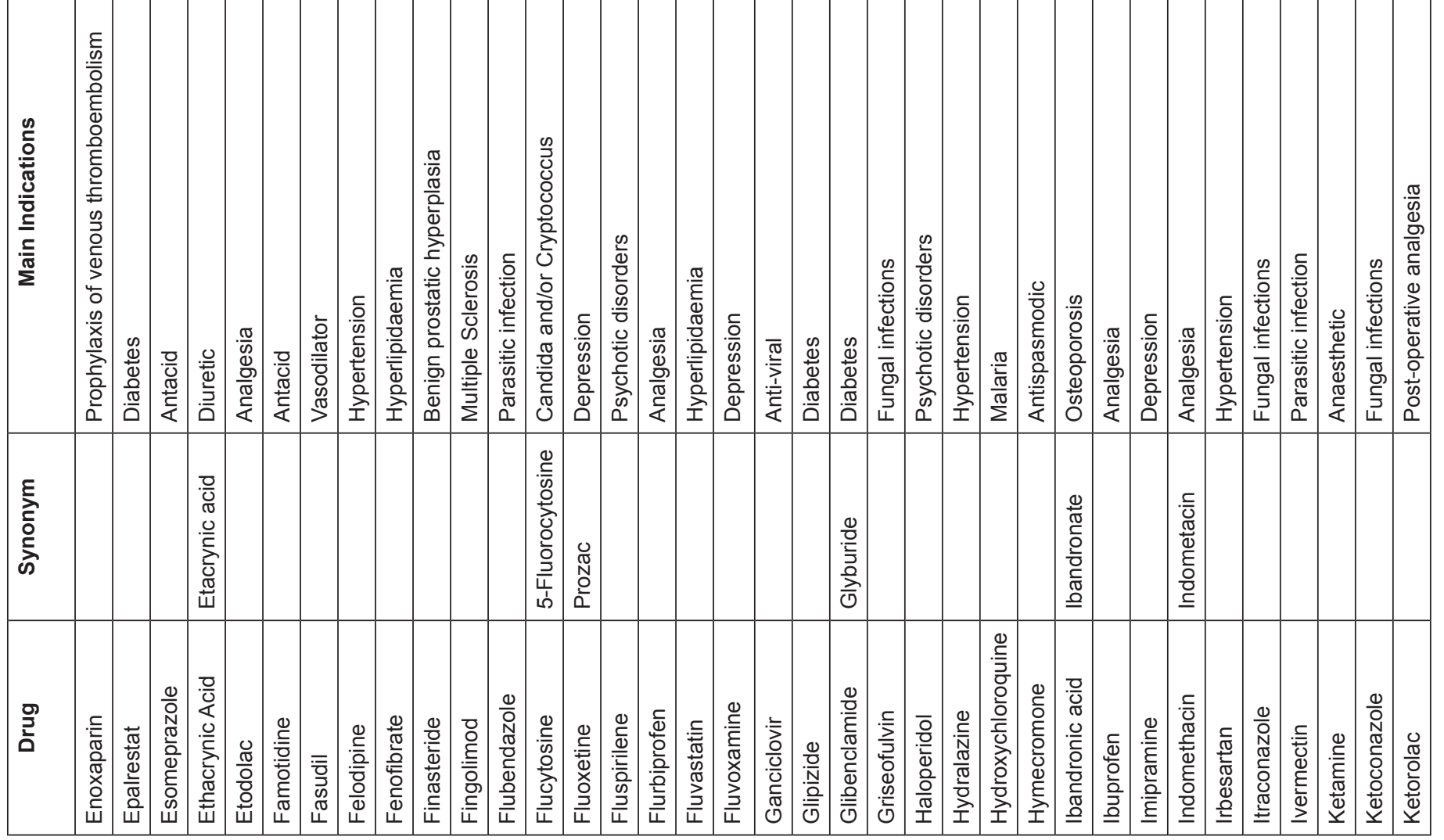


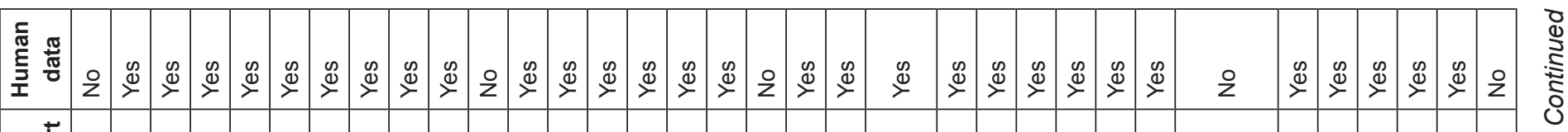

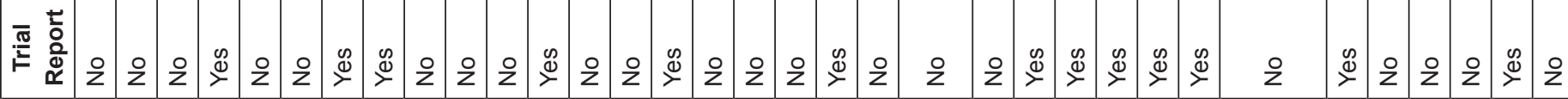

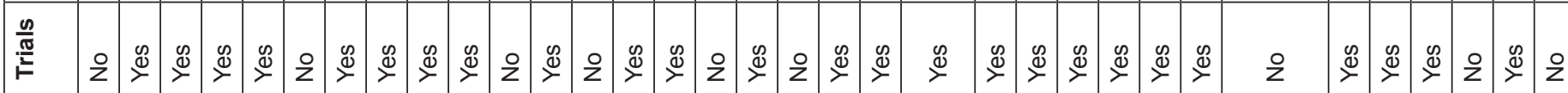
离 戠

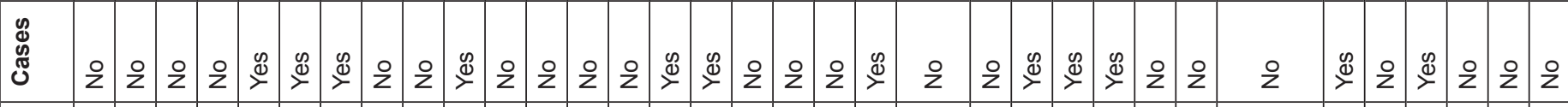

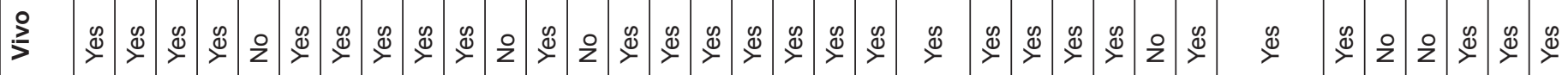
竞

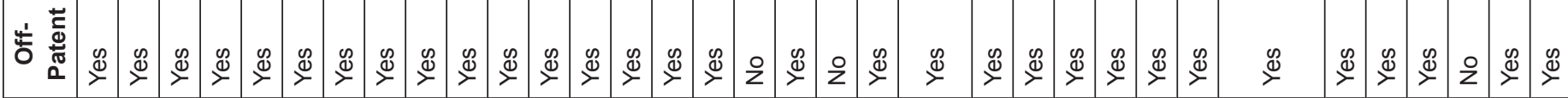

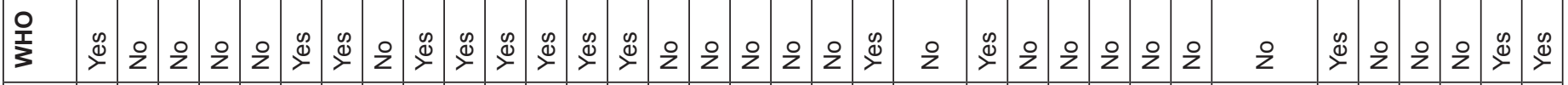

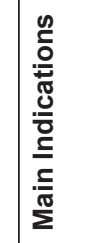

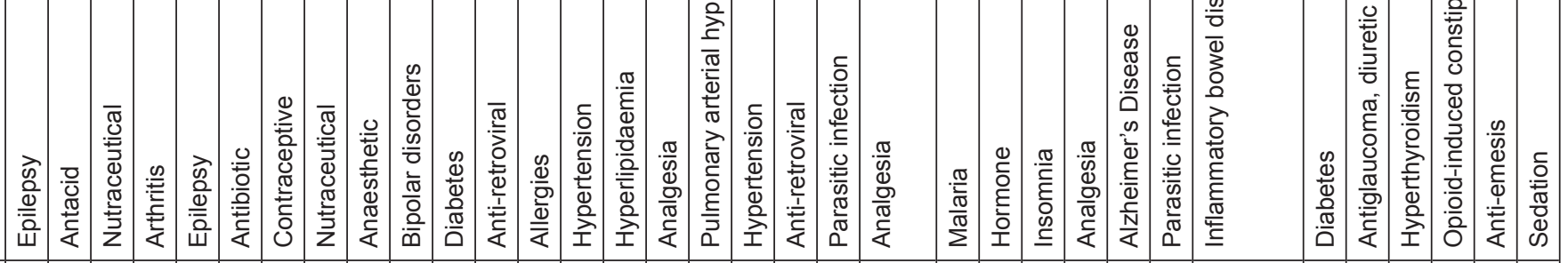
紊

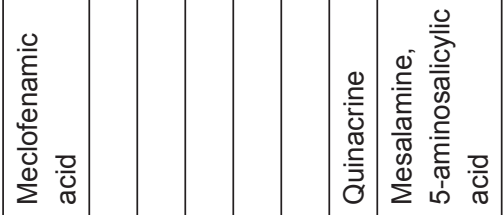

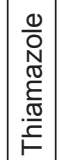

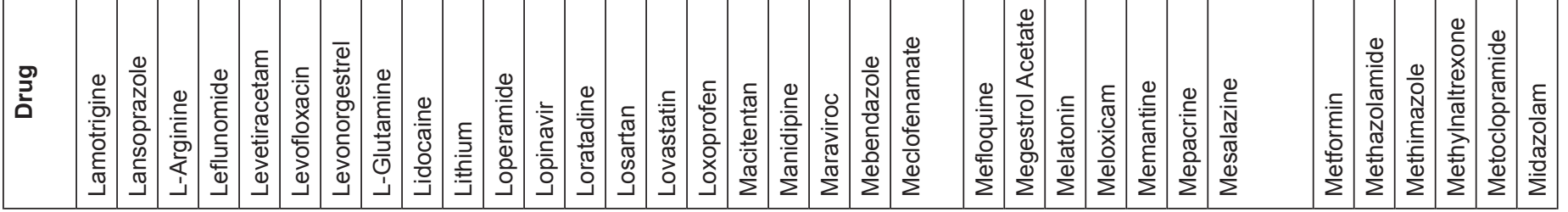




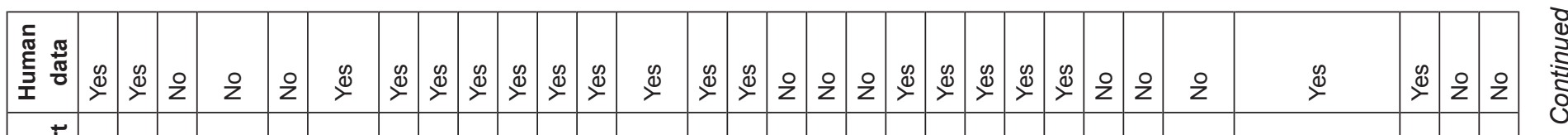

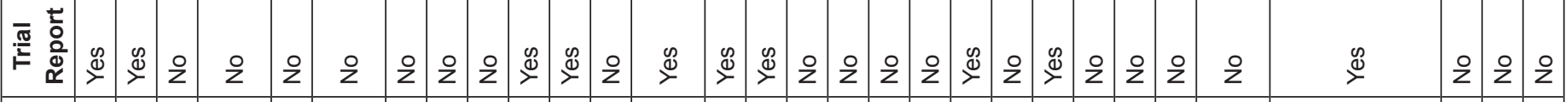

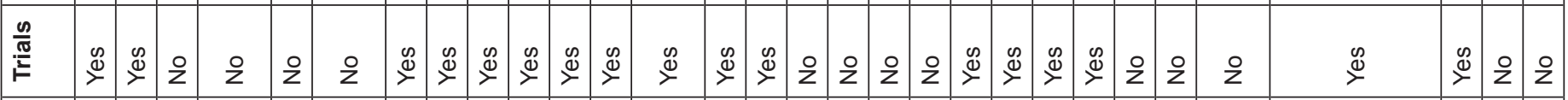

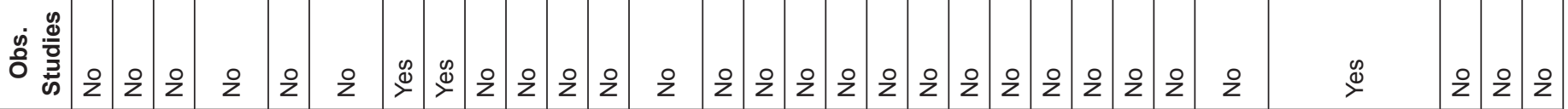

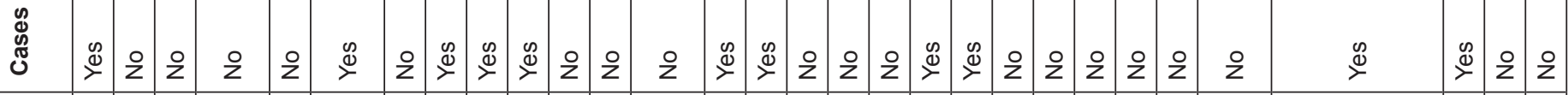

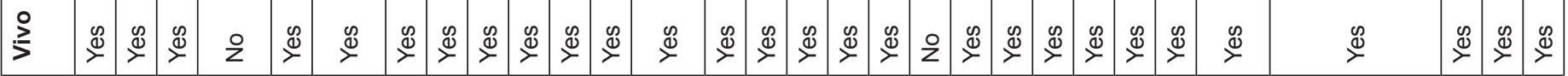

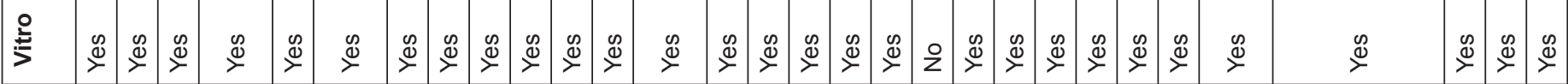

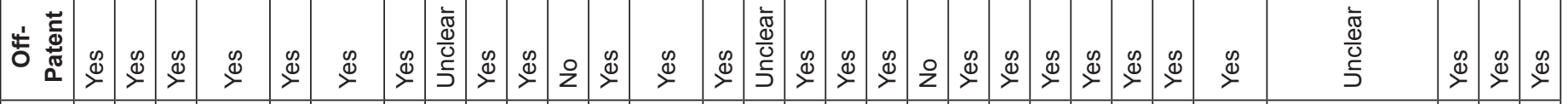

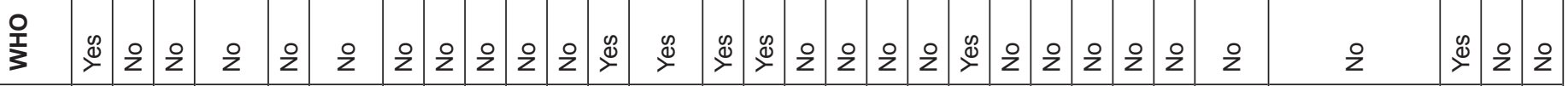

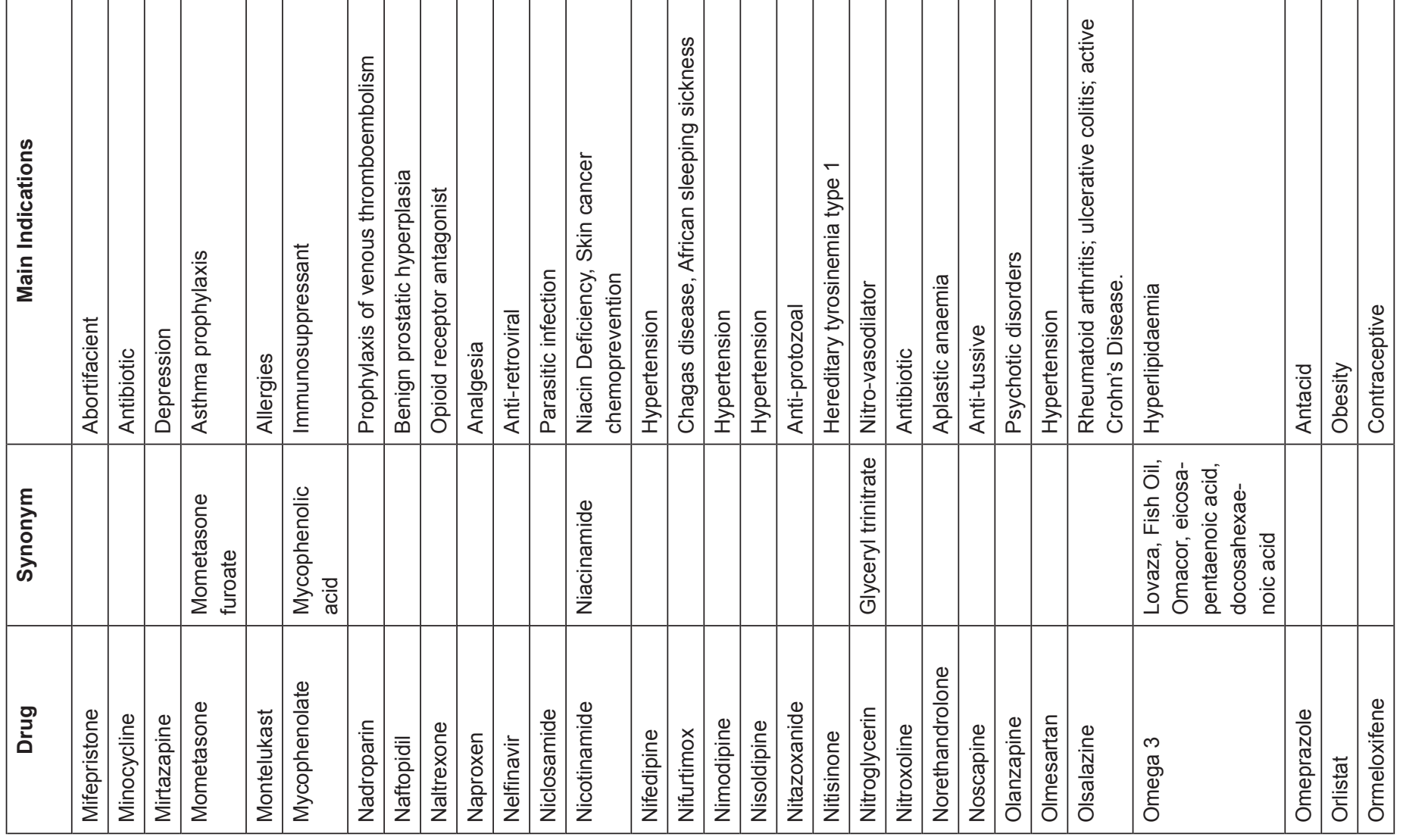




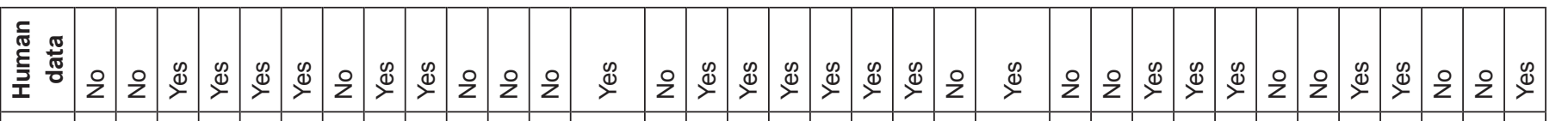

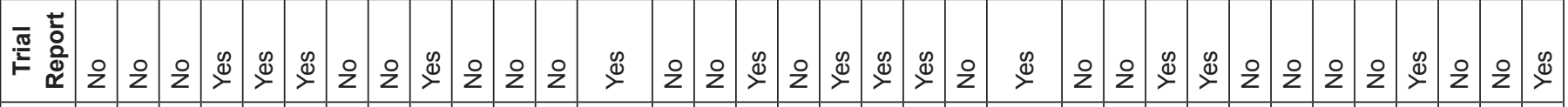

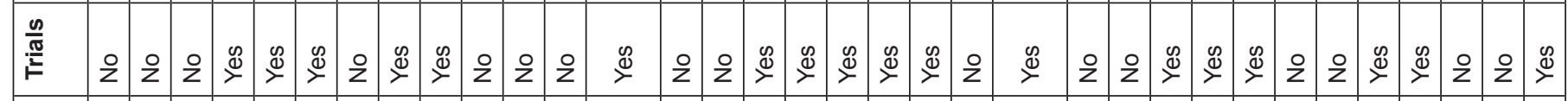

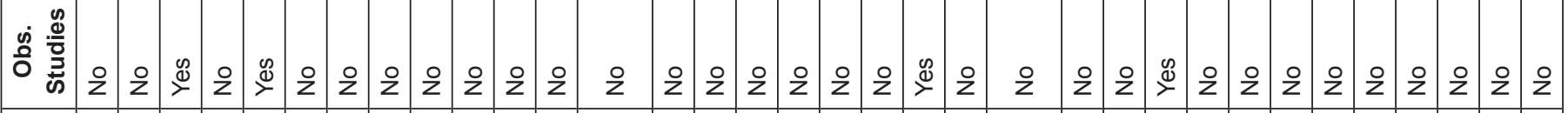

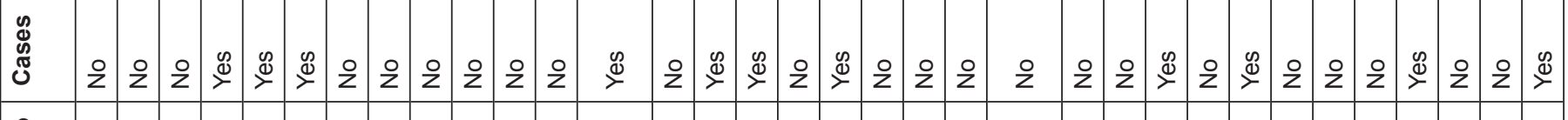

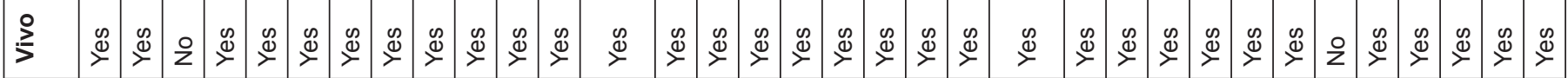

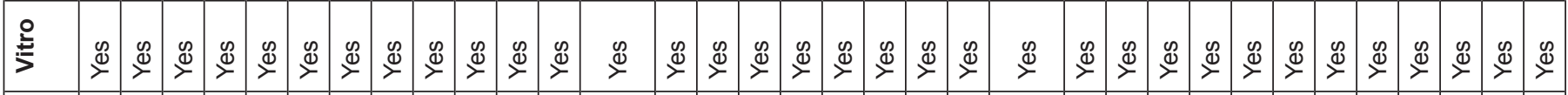

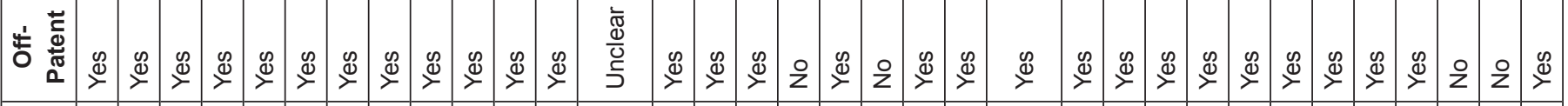

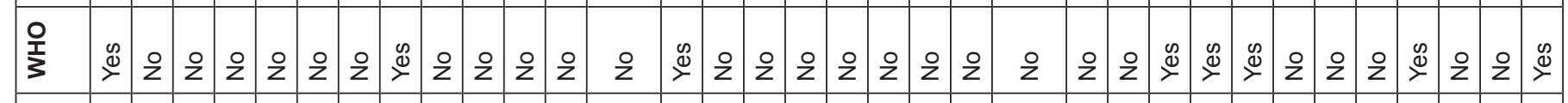

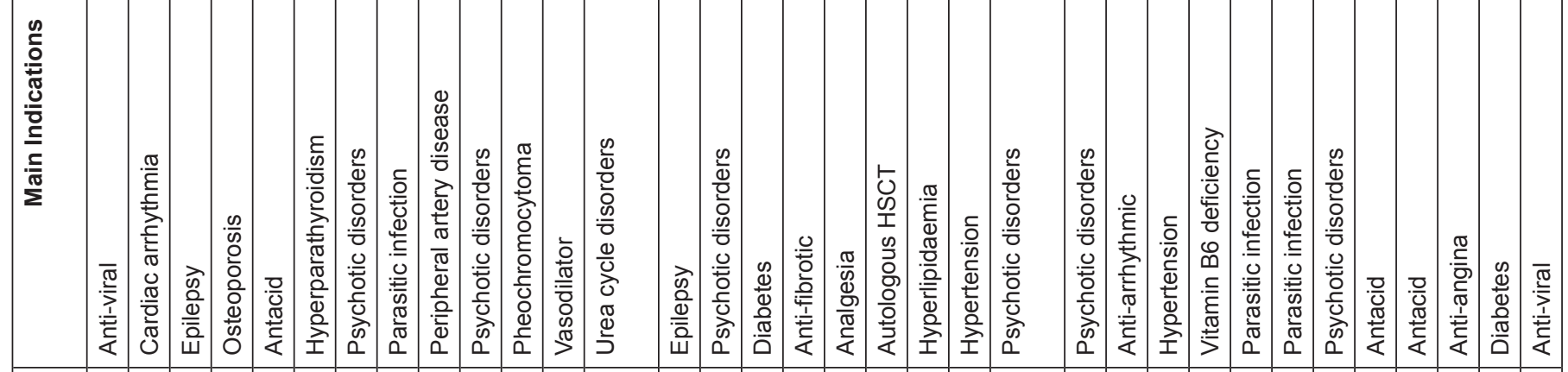

\begin{tabular}{|c|c|c|c|c|c|c|c|c|c|c|c|c|c|c|c|c|c|c|c|c|c|c|c|c|c|c|c|c|c|c|c|c|c|c|}
\hline 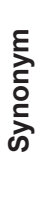 & & & & 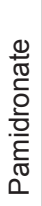 & & 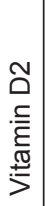 & & & & & & & 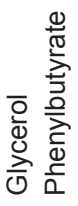 & & & & & & $\begin{array}{l}\frac{8}{\circ} \\
\stackrel{\operatorname{m}}{2} \\
\stackrel{2}{<}\end{array}$ & & & 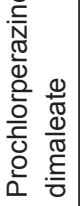 & & & & 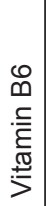 & & 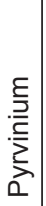 & & & & & & \\
\hline 올 & 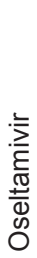 & 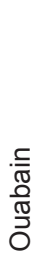 & 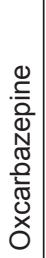 & 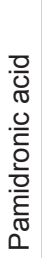 & $\begin{array}{l}\frac{1}{0} \\
\text { N } \\
\text { तై } \\
\frac{0}{0} \\
\frac{1}{0} \\
\text { ர } \\
0\end{array}$ & 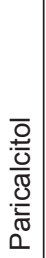 & $\begin{array}{l}\overline{0} \\
\frac{0}{2} \\
\underline{3} \\
\frac{1}{c} \\
\overline{0} \\
0\end{array}$ & 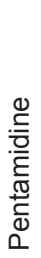 & 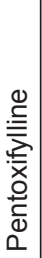 & 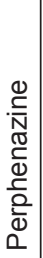 & 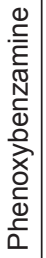 & 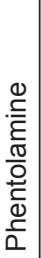 & 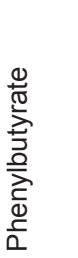 & $\begin{array}{l}\frac{c}{\overline{0}} \\
\frac{\operatorname{c}}{\mathbb{C}} \\
\frac{\mathbb{d}}{\square}\end{array}$ & 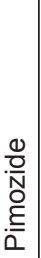 & 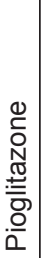 & 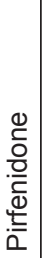 & 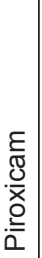 & $\begin{array}{l}\frac{1}{0} \\
\frac{0}{2} \\
\frac{x}{2} \\
\frac{0}{2}\end{array}$ & 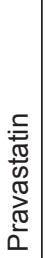 & 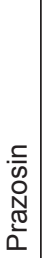 & 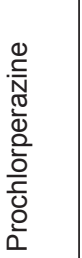 & 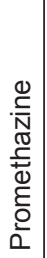 & 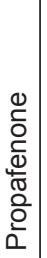 & $\begin{array}{l}\overline{ } \\
\text { 을 } \\
\text { 즌 } \\
\text { 응 } \\
\text { 으 }\end{array}$ & 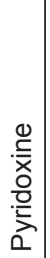 & 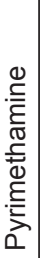 & 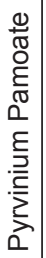 & 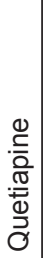 & 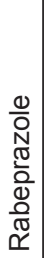 & 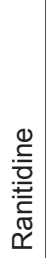 & 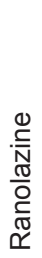 & 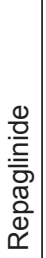 & 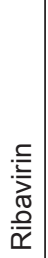 \\
\hline
\end{tabular}




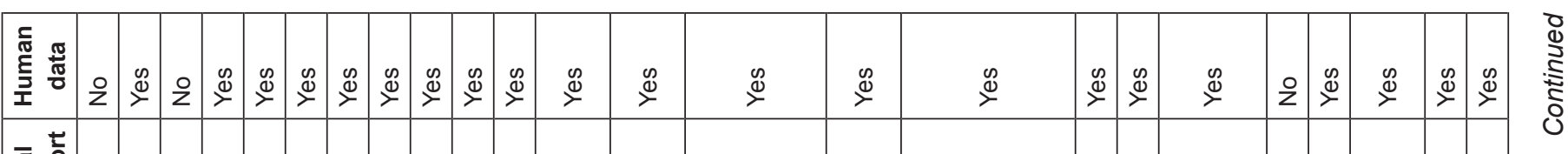

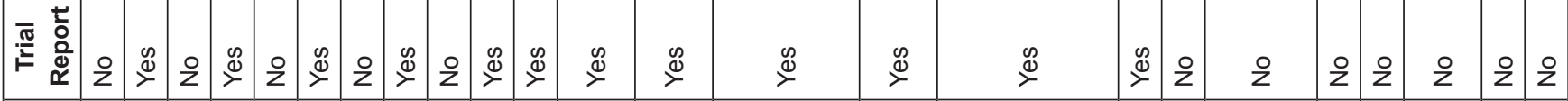

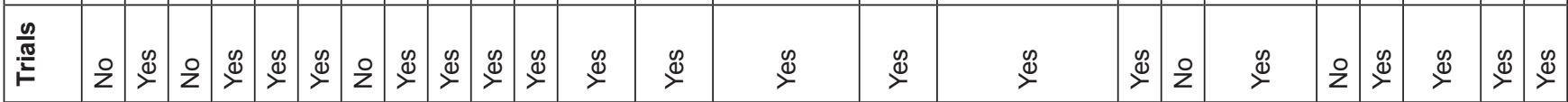
离产离

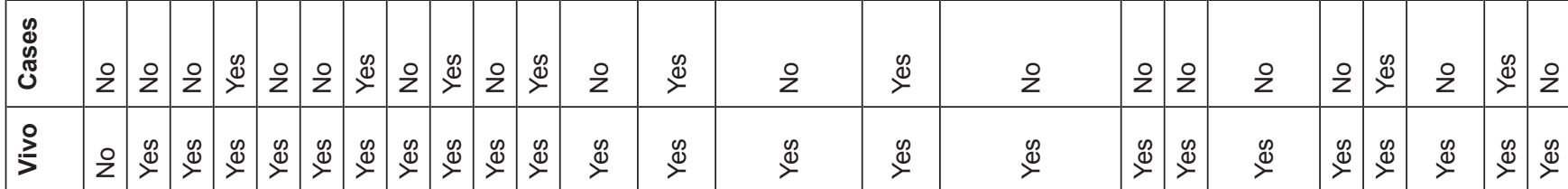

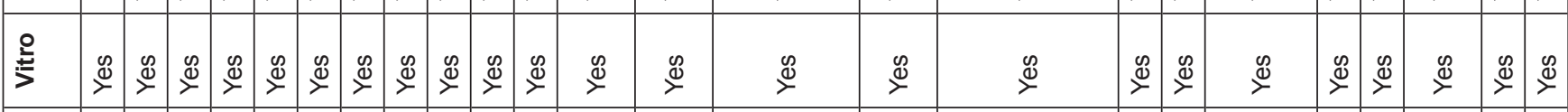

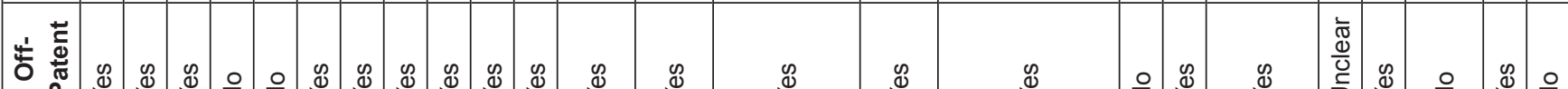

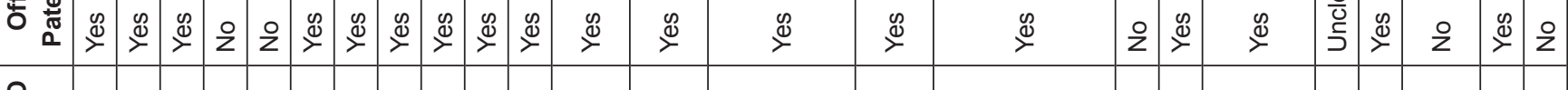

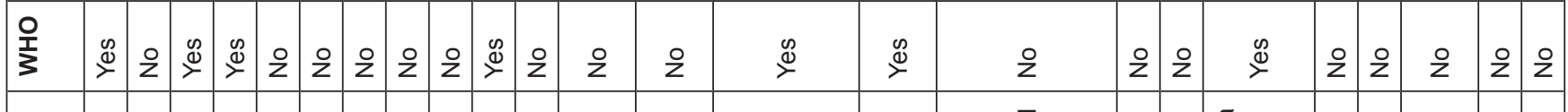

\begin{tabular}{|c|c|c|c|c|c|c|c|c|c|c|c|c|c|c|c|c|c|c|c|}
\hline 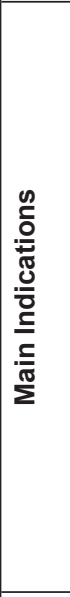 & 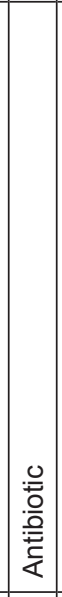 & & 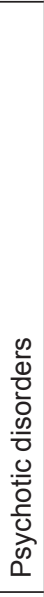 & 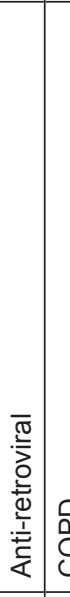 & 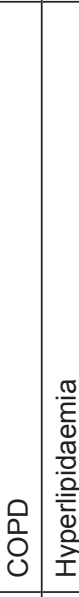 & 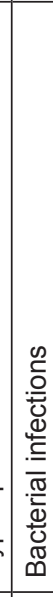 & 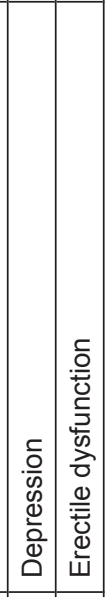 & 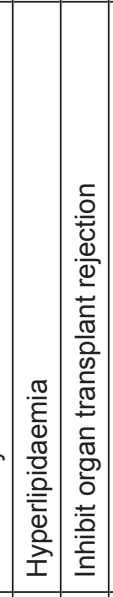 & 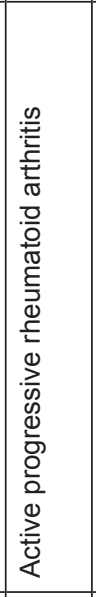 & 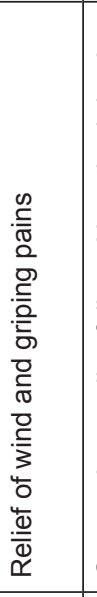 & 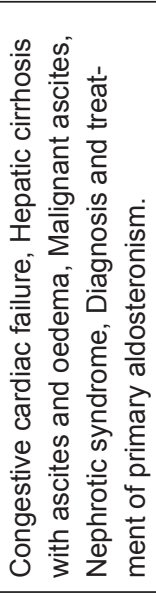 & 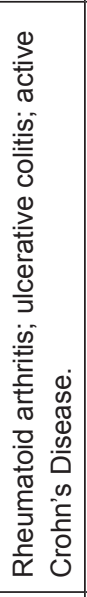 & 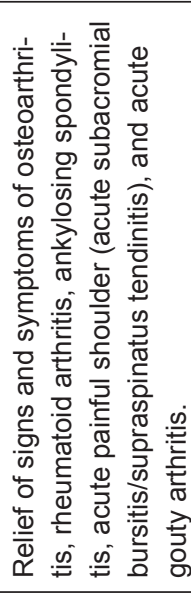 & 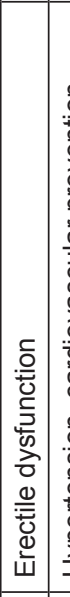 & 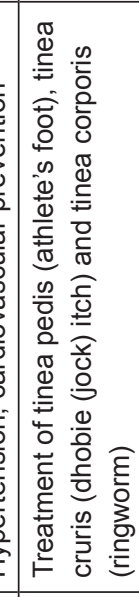 & 苞 & 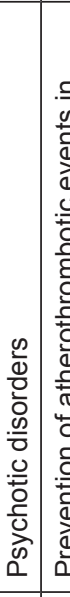 & 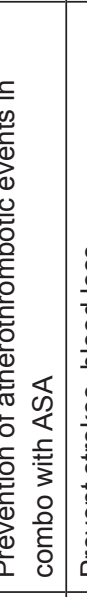 & 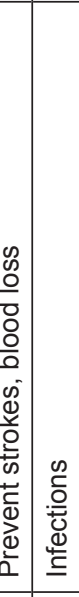 \\
\hline 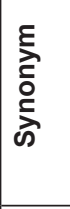 & & & & & & & & 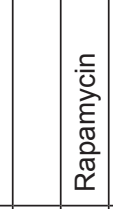 & & & & & & & & & & & \\
\hline 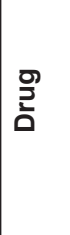 & & & & $=$ & 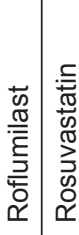 & 10 & 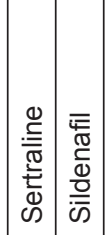 & 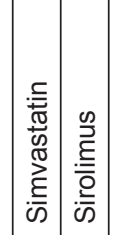 & 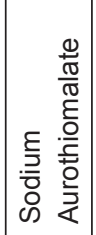 & 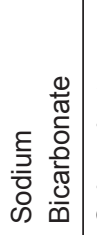 & 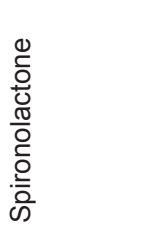 & 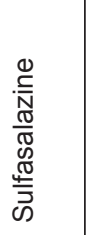 & 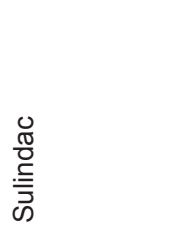 & 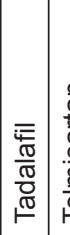 & 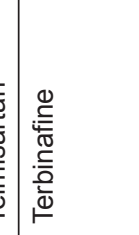 & 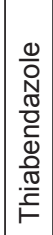 & & & 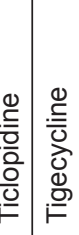 \\
\hline
\end{tabular}




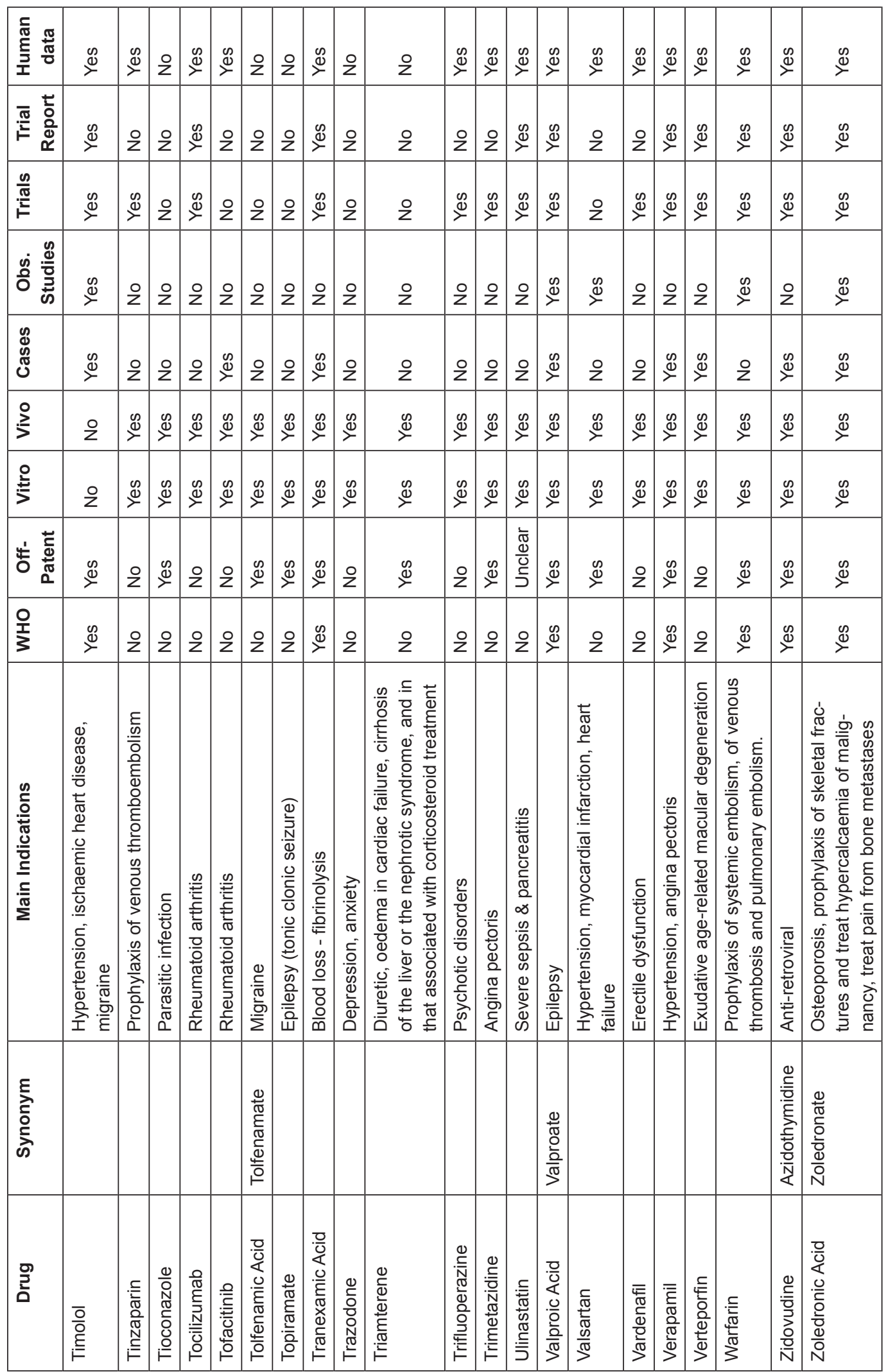

